\title{
Global Attractors for the Three-Dimensional Viscous Primitive Equations of Large-Scale Atmosphere in Log-Pressure Coordinate
}

\author{
Bo You and Shan Ma \\ School of Mathematics and Statistics, Lanzhou University, Lanzhou, Gansu 730000, China \\ Correspondence should be addressed to Bo You; youb03@126.com
}

Received 4 September 2012; Revised 14 December 2012; Accepted 21 December 2012

Academic Editor: Grzegorz Lukaszewicz

Copyright (c) 2013 B. You and S. Ma. This is an open access article distributed under the Creative Commons Attribution License, which permits unrestricted use, distribution, and reproduction in any medium, provided the original work is properly cited.

We will first prove the existence of $(V, V)$ - and $\left(V, H^{2}\right)$-absorbing sets for the three-dimensional primitive equations of large-scale atmosphere in log-pressure coordinate and then prove the existence of $\left(V, H^{3}\right)$-absorbing set by the use of the elliptic regularity theory. Finally, we obtain the existence of $(V, V)$ - and $\left(V, H^{2}\right)$-global attractors for the three-dimensional viscous primitive equations of large-scale atmosphere in log-pressure coordinate by using the Sobolev compactness embedding theory.

\section{Introduction}

The fundamental equations governing the motion of the atmosphere consist of the Navier-Stokes equations with Coriolis force and thermodynamics, which account for the buoyancy forces and stratification effects under the Boussinesq approximation. Moreover, due to the shallowness of the atmosphere, that is, the depth of the fluid layer is very small in comparison with the radius of the earth, the vertical large-scale motion in the atmosphere is much smaller than the horizontal one, which in turn leads to modeling the vertical motion by hydrostatic balance [1-3]. But for largescale atmospheric flows, it is often necessary to take account of compressibility. This may be done most easily by a change of vertical coordinates to log-pressure coordinate rather than a function of geometric height. As a result, one can obtain the system (1)-(4), which is known as the primitive equations for large-scale atmosphere in log-pressure coordinate.

In recent years, the primitive equations of the atmosphere, the ocean, and the coupled atmosphere-ocean have been extensively studied from the mathematical point of view $[1,4-$ 13]. The mathematical framework of the primitive equations of the ocean was formulated, and the existence of weak solutions was proved by Lions et al. in [11]. In [1], the authors have studied the primitive equations of large-scale atmosphere in pressure coordinate, where the continuity equation is changed into the incompressible equation, but the thermodynamical equation is very complicated. For the sake of simplicity, the authors have made some approximation of the thermodynamical equation, and in this case, the authors have proved the existence and the uniqueness of strong, localin-time solutions. By assuming that the initial data is small enough, the authors have studied the existence of strong, global-in-time solutions to the three-dimensional primitive equations of large-scale ocean and obtained the existence of strong, local-in-time solutions to the equations for all initial data in [14]. In [5], the authors have proved the maximum principles of the temperature for the primitive equations of the atmosphere in pressure coordinate. The existence and the uniqueness of strong, global-in-time solutions to the primitive equations in thin domains for a large set of initial datum whose sizes depend inversely on the thickness were established in [9]. In [15], Temam and Ziane have considered the existence of strong, local-in-time solutions for the primitive equations of the atmosphere, the ocean, and the coupled atmosphere-ocean. Asymptotic analysis of the primitive equations under the small depth assumption was established in [10]. In [8], the authors have proved the existence of weak solutions and a trajectory attractors for the moist atmospheric equations in geophysics. The existence and the uniqueness 
of strong, global-in-time solutions for the three-dimensional viscous primitive equations of large-scale ocean and atmosphere dynamics were established by the authors in $[4,16]$. In [6], the authors have considered the long-time dynamics of the primitive equations of large-scale atmosphere and obtained a weakly compact global attractor $\mathscr{A}$ which captures all the trajectories with respect to the weak topology of $V$. The existence and the uniqueness of strong solutions, the existence of smooth solutions, and the existence of a compact global attractor in $V$ for the primitive equations with more regular initial data than that in [6] were established in [7]. In [12], the authors have proved the existence and the uniqueness of $z$-weak, global-in-time solutions when the initial conditions satisfy some regularity. The existence of global attractor in $V$ for the primitive equations with initial datum in $V$ and the heat source $Q \in L^{2}(\Omega)$ was proved by use of the Aubin-Lions compactness lemma in [17]. In [18], the authors have proved the existence of the global attractor in $\left(H^{2}(\Omega)\right)^{3}$ for the three-dimensional viscous primitive equations of large-scale ocean and atmosphere dynamics. In this paper, we will consider the primitive equations of large-scale atmosphere in log-pressure coordinate, where the continuity equation is transformed into the incompressible equation with the weighted function $e^{-z / H_{s}}$, which is more complicated than that in pressure coordinate, but we do not need to make any approximation of the thermodynamical equation such that the accuracy of the temperature is higher than that in pressure coordinate which will reveal the real world better. Moreover, the appearance of the weighted function $e^{-z / H_{s}}$ makes the calculations more difficult. In order to make sure of the existence of the absorbing set, we need to add some additional conditions (10) on $h$, which means that the thickness of $\Omega$ is very small. In [18], there are not any restrictions on the thickness of $\Omega$ to ensure the existence of the absorbing sets, and the $H^{1}(\Omega)$ estimate of $T$ can be obtained directly by taking the inner product (15) with $-\Delta_{3} T$. But in this paper, thanks to the appearance of the weighted function $e^{-z / H_{s}}$, we have to first estimate $T_{z}$ in $L^{2}$ and then $\nabla_{2} T$ in $L^{2}$ to obtain a priori estimates on $T$ in $H^{1}$. In this paper, we will prove the existence of $\left(V, H^{2}\right)$-global attractor for the three-dimensional primitive equations of large-scale atmosphere in log-pressure coordinate under the assumptions (10) on $h$.

In this paper, we will consider the following threedimensional viscous primitive equations of large-scale atmosphere in log-pressure coordinate [3]:

$$
\begin{gathered}
\frac{\partial v}{\partial t}+(v \cdot \nabla) v+w \frac{\partial v}{\partial z}+f_{0} \vec{k} \times v+\nabla \Phi+L_{1} v=0 \\
\frac{\partial \Phi}{\partial z}=\frac{R T}{H_{s}} \\
\nabla \cdot\left(e^{-z / H_{s}} v\right)+\frac{\partial}{\partial z}\left(e^{-z / H_{s}} w\right)=0 \\
\frac{\partial T}{\partial t}+v \cdot \nabla T+w \frac{\partial T}{\partial z}+L_{2} T=Q
\end{gathered}
$$

in the domain

$$
\Omega=M \times(-h, 0) \subset \mathbb{R}^{3},
$$

where $M$ is a bounded domain in $\mathbb{R}^{2}$ with smooth boundary. Here, $v=\left(v_{1}, v_{2}\right),\left(v_{1}, v_{2}, w\right)$ is the velocity field, $T$ is the temperature, $\Phi$ is the geopotential, $f_{0}=2 \Omega_{1} \sin v_{0}$ is the Coriolis parameter, $\vec{k}$ is the vertical unit vector, $R$ is a positive constant, and $Q$ is the heat source. The operators $L_{1}$ and $L_{2}$ are given by

$$
\begin{aligned}
& L_{1}=-\frac{1}{\mathrm{Re}_{1}} \Delta-\frac{1}{\mathrm{Re}_{2}} \frac{\partial^{2}}{\partial z^{2}}, \\
& L_{2}=-\frac{1}{R t_{1}} \Delta-\frac{1}{R t_{2}} \frac{\partial^{2}}{\partial z^{2}},
\end{aligned}
$$

where $\mathrm{Re}_{1}$ and $\mathrm{Re}_{2}$ are positive constants representing the horizontal and the vertical Reynolds numbers, respectively, and $R t_{1}$ and $R t_{2}$ are positive constants which stand for the horizontal and the vertical heat diffusivities, respectively. And $z=-H_{s} \log \left(p / p_{*}\right)$ is used as a vertical coordinate, where $H_{s}$ is a constant "scale height" and $p_{*}$ is a constant reference pressure. For the sake of simplicity, let $\nabla=\left(\partial_{x}, \partial_{y}\right)$ be the horizontal gradient operator and $\Delta=\partial_{x}^{2}+\partial_{y}^{2}$ the horizontal Laplacian.

We denote the different parts of the boundary $\partial \Omega$ by

$$
\begin{aligned}
\Gamma_{u} & =\{(x, y, z) \in \bar{\Omega}: z=0\}, \\
\Gamma_{b} & =\{(x, y, z) \in \bar{\Omega}: z=-h\}, \\
\Gamma_{l} & =\{(x, y, z) \in \bar{\Omega}:(x, y) \in \partial M,-h \leq z \leq 0\} .
\end{aligned}
$$

We equip (1)-(4) with the following boundary conditions, with nonslip and nonflux on the side walls and bottom [4]:

$$
\begin{gathered}
\left.\frac{\partial v}{\partial z}\right|_{\Gamma_{u}}=0,\left.\quad w\right|_{\Gamma_{u}}=0,\left.\quad\left(\frac{1}{R t_{2}} \frac{\partial T}{\partial z}+\alpha T\right)\right|_{\Gamma_{u}}=0, \\
\left.\frac{\partial v}{\partial z}\right|_{\Gamma_{b}}=0,\left.\quad w\right|_{\Gamma_{b}}=0,\left.\quad \frac{\partial T}{\partial z}\right|_{\Gamma_{b}}=0, \\
\left.v \cdot \vec{n}\right|_{\Gamma_{l}}=0, \quad \frac{\partial v}{\partial \vec{n}} \times\left.\vec{n}\right|_{\Gamma_{l}}=0,\left.\quad \frac{\partial T}{\partial \vec{n}}\right|_{\Gamma_{l}}=0,
\end{gathered}
$$

where $\alpha$ is a positive constant.

In addition, (1)-(8) are supplemented with the following initial conditions:

$$
\begin{aligned}
v(x, y, z, 0) & =v_{0}(x, y, z), \\
T(x, y, z, 0) & =T_{0}(x, y, z) .
\end{aligned}
$$

Additionally, assume that $h$ satisfy

$$
\begin{gathered}
\frac{9 h^{2}}{4 H_{s}^{2}}+\frac{4 h}{\alpha H_{s}^{2} R t_{2}}<1, \\
H_{s}^{2} \operatorname{Re}_{2} \lambda>1 .
\end{gathered}
$$


This paper is organized as follows. Section 2 is devoted to introduce the mathematical framework and the definition of solutions for (1)-(10) and give some auxiliary lemmas used in the sequel. In Section 3, we will give the existence and the uniqueness of solutions for (1)-(10). Next, some a priori estimates of solutions corresponding to (1)-(10) will be established, which imply the existence of $(V, V)$ - and $\left(V, H^{2}\right)$ absorbing sets for (1)-(10). In Section 4, we first obtain the existence of the $(V, V)$-global attractor for (1)-(10) by $H^{2} \subset V$ compactly, and then get the existence of $\left(V, H^{3}\right)$-absorbing set of (1)-(10) by the elliptic regularity theory. Finally, we prove the existence of the $\left(V, H^{2}\right)$-global attractor for the three-dimensional viscous primitive equations of large-scale atmosphere in log-pressure coordinate by the Sobolev compactness embedding theory.

Throughout this paper, let $C$ be positive constants independent of $t$ which may be different from line to line.

\section{Mathematical Setting of (1)-(10)}

2.1. Reformulation of (1)-(9). Integrating the continuity equation (3) and taking the boundary conditions for $w$ into account, we obtain

$$
\begin{gathered}
w(x, y, z, t)=-\int_{-h}^{z} e^{(z-\zeta) / H_{s}} \nabla \cdot v(x, y, \zeta, t) d \zeta, \\
\int_{-h}^{0} e^{-\zeta / H_{s}} \nabla \cdot v(x, y, \zeta, t) d \zeta=0 .
\end{gathered}
$$

And then, we consider the vertical momentum equation, that is, the second equation (2). First, we define an unknown function on $z=0$ (i.e., $p=p_{*}$ ), where $p_{*}$ is a constant reference pressure, say

$$
\Phi_{s}: M \longrightarrow \mathbb{R},
$$

which is the geopotential of the atmosphere on $z=0$.

Then,

$$
\Phi(x, y, z, t)=\Phi_{s}(x, y, t)+\frac{R}{H_{s}} \int_{0}^{z} T(x, y, \zeta, t) d \zeta
$$

Therefore, (1)-(4) can be rewritten as follows:

$$
\begin{aligned}
\frac{\partial v}{\partial t}+ & (v \cdot \nabla) v-\left(\int_{-h}^{z} e^{(z-\zeta) / H_{s}} \nabla \cdot v(x, y, \zeta, t) d \zeta\right) \frac{\partial v}{\partial z} \\
& +\nabla \Phi_{s}(x, y, t)+f_{0} \vec{k} \times v \\
& +\frac{R}{H_{s}} \int_{0}^{z} \nabla T(x, y, \zeta, t) d \zeta+L_{1} v=0, \\
\frac{\partial T}{\partial t}+v \cdot \nabla T & -\left(\int_{-h}^{z} e^{(z-\zeta) / H_{s}} \nabla \cdot v(x, y, \zeta, t) d \zeta\right) \frac{\partial T}{\partial z}+L_{2} T=Q,
\end{aligned}
$$

with the following boundary conditions:

$$
\begin{gathered}
\left.\frac{\partial v}{\partial z}\right|_{\Gamma_{b}}=0,\left.\quad \frac{\partial v}{\partial z}\right|_{\Gamma_{u}}=0 \\
\left.v \cdot \vec{n}\right|_{\Gamma_{l}}=0, \quad \frac{\partial v}{\partial \vec{n}} \times\left.\vec{n}\right|_{\Gamma_{l}}=0, \\
\left.\frac{\partial T}{\partial z}\right|_{\Gamma_{b}}=0,\left.\quad\left(\frac{1}{R t_{2}} \frac{\partial T}{\partial z}+\alpha T\right)\right|_{\Gamma_{u}}=0,\left.\quad \frac{\partial T}{\partial \vec{n}}\right|_{\Gamma_{l}}=0,
\end{gathered}
$$

and the initial data

$$
\begin{gathered}
v(x, y, z, 0)=v_{0}(x, y, z), \\
T(x, y, z, 0)=T_{0}(x, y, z) .
\end{gathered}
$$

At last, we will divide (14) into two systems with respect to $\bar{v}$ and $\widetilde{v}$ [4], where $\bar{v}$ and $\widetilde{v}$ are defined by

$$
\begin{gathered}
\bar{v}=\frac{1}{A} \int_{-h}^{0} e^{-z / H_{s}} v(x, y, z) d z, \\
\widetilde{v}=v-\bar{v},
\end{gathered}
$$

where

$$
A=\int_{-h}^{0} e^{-z / H_{s}} d z=H_{s}\left(e^{h / H_{s}}-1\right),
$$

and we have $\overline{\widetilde{v}}=0$.

Therefore, $\bar{v}$ satisfies the following systems:

$$
\begin{gathered}
\frac{\partial \bar{v}}{\partial t}+(\bar{v} \cdot \nabla) \bar{v}-\frac{1}{\operatorname{Re}_{1}} \Delta \bar{v}+\nabla \Phi_{s}(x, y, t)+\overline{(\nabla \cdot \widetilde{v}) \widetilde{v}+(\widetilde{v} \cdot \nabla) \widetilde{v}} \\
+f_{0} \vec{k} \times \bar{v}+\frac{R}{\frac{R}{H_{s}} \int_{0}^{z} \nabla T(x, y, \zeta, t) d \zeta-\frac{1}{H_{s} \operatorname{Re}_{2}} \frac{\partial \widetilde{v}}{\partial z}}=0,
\end{gathered}
$$

with the boundary conditions

$$
\nabla \cdot \bar{v}=0,\left.\quad \bar{v} \cdot \vec{n}\right|_{\Gamma_{l}}=0, \quad \frac{\partial \bar{v}}{\partial \vec{n}} \times\left.\vec{n}\right|_{\Gamma_{l}}=0,
$$

and $\widetilde{v}$ satisfies the following systems

$$
\begin{aligned}
\frac{\partial \widetilde{v}}{\partial t} & (\widetilde{v} \cdot \nabla) \widetilde{v}-\left(\int_{-h}^{z} e^{(z-\zeta) / H_{s}} \nabla \cdot \widetilde{v}(x, y, \zeta, t) d \zeta\right) \frac{\partial \widetilde{v}}{\partial z} \\
& +\frac{R}{H_{s}} \int_{0}^{z} \nabla T(x, y, \zeta, t) d \zeta+f_{0} \vec{k} \times \widetilde{v}+L_{1} \widetilde{v}+(\widetilde{v} \cdot \nabla) \bar{v}+(\bar{v} \cdot \nabla) \widetilde{v} \\
& -\overline{(\nabla \cdot \widetilde{v}) \widetilde{v}+(\widetilde{v} \cdot \nabla) \widetilde{v}+\frac{R}{H_{s}} \int_{0}^{z} \nabla T(x, y, \zeta, t) d \zeta-\frac{1}{H_{s} \operatorname{Re}_{2}} \frac{\partial \widetilde{v}}{\partial z}}=0,
\end{aligned}
$$

with the boundary conditions

$$
\begin{aligned}
\left.\frac{\partial \widetilde{v}}{\partial z}\right|_{\Gamma_{u}}=0, & \left.\frac{\partial \widetilde{v}}{\partial z}\right|_{\Gamma_{b}}=0,\left.\quad \widetilde{v} \cdot \vec{n}\right|_{\Gamma_{l}}=0, \\
& \frac{\partial \widetilde{v}}{\partial \vec{n}} \times\left.\vec{n}\right|_{\Gamma_{l}}=0 .
\end{aligned}
$$


2.2. Some Function Spaces. In this subsection, we will quote briefly some notations and function spaces used in this paper.

First of all, we introduce the notations for some function spaces on $\Omega$ as follows:

$$
\begin{gathered}
\mathscr{V}_{1}=\left\{v \in\left(C^{\infty}(\bar{\Omega})\right)^{2}:\left.\frac{\partial v}{\partial z}\right|_{\Gamma_{u}}=0,\left.\frac{\partial v}{\partial z}\right|_{\Gamma_{b}}=0,\left.v \cdot \vec{n}\right|_{\Gamma_{l}}=0,\right. \\
\left.\quad \frac{\partial v}{\partial \vec{n}} \times\left.\vec{n}\right|_{\Gamma_{l}}=0, \int_{-h}^{0} e^{-z / H_{s}} \nabla \cdot v(x, y, z) d z=0\right\}, \\
\mathscr{V}_{2}=\left\{T \in C^{\infty}(\bar{\Omega}):\left.\frac{\partial T}{\partial z}\right|_{\Gamma_{b}}=0,\right. \\
\left.\left.\left(\frac{1}{R t_{2}} \frac{\partial T}{\partial z}+\alpha T\right)\right|_{\Gamma_{u}}=0,\left.\frac{\partial T}{\partial \vec{n}}\right|_{\Gamma_{l}}=0\right\}
\end{gathered}
$$

Let $L^{p}$ (or $H^{s}$ ) be the weighted Hilbert spaces of $L^{p}$ (or $H^{s}$ ) functions or $L^{p}$ (or $H^{s}$ )-vector valued functions on $\Omega$ and denote the usual $L^{p}(\Omega)$-norms by $\|\cdot\|_{L^{p}(\Omega)}$, where $1 \leq p \leq$ $\infty$ (or $s \geq 0)$. Denote the inner product in $L^{2}$ by $(\cdot, \cdot)$ and the norm in $L^{p}$ by $\|\cdot\|_{p}$, respectively, given by

$$
\begin{gathered}
\left(h_{1}, h_{2}\right)=\int_{\Omega} e^{-z / H_{s}} h_{1} \cdot h_{2} d x d y d z, \\
\|h\|_{p}^{p}=\int_{\Omega} e^{-z / H_{s}}|h|^{p} d x d y d z,
\end{gathered}
$$

for any $h_{1}, h_{2} \in L^{2}$ and $h \in L^{p}(1 \leq p \leq \infty)$.

Similarly, define

$$
\begin{aligned}
\left(\left(v_{1}, v_{2}\right)\right)= & \frac{1}{\operatorname{Re}_{1}} \int_{\Omega} e^{-z / H_{s}} \nabla v_{1} \cdot \nabla v_{2} d x d y d z \\
& +\frac{1}{\operatorname{Re}_{2}} \int_{\Omega} e^{-z / H_{s}} \frac{\partial v_{1}}{\partial z} \cdot \frac{\partial v_{2}}{\partial z} d x d y d z, \\
\left(\left(T_{1}, T_{2}\right)\right)= & \frac{1}{R t_{1}} \int_{\Omega} e^{-z / H_{s}} \nabla T_{1} \cdot \nabla T_{2} d x d y d z \\
& +\frac{1}{R t_{2}} \int_{\Omega} e^{-z / H_{s}} \frac{\partial T_{1}}{\partial z} \frac{\partial T_{2}}{\partial z} d x d y d z \\
& +\alpha \int_{\Gamma_{u}} T_{1} T_{2} d x d y
\end{aligned}
$$

for any $v_{1}, v_{2} \in \mathscr{V}_{1}$ and $T_{1}, T_{2} \in \mathscr{V}_{2}$, respectively.

Let $V_{1}, V_{2}$ be the closure of $\mathscr{V}_{1}, \mathscr{V}_{2}$ with respect to the following norms:

$$
\|v\|^{2}=((v, v)), \quad\|T\|^{2}=((T, T)),
$$

for any $v \in \mathscr{V}_{1}$ and $T \in \mathscr{V}_{2}$, respectively, and let $V=V_{1} \times V_{2}$.

Next, we recall some results used in the sequel.
Lemma 1 (see [19]). (i) for $u \in H^{1}(M)$,

$$
\begin{aligned}
& \|u\|_{L^{4}(M)} \leq C\|u\|_{L^{2}(M)}^{1 / 2}\|u\|_{H^{1}(M)}^{1 / 2}, \\
& \|u\|_{L^{6}(M)} \leq C\|u\|_{L^{2}(M)}^{2 / 3}\|u\|_{H^{1}(M)}^{1 / 3}, \\
& \|u\|_{L^{8}(M)} \leq C\|u\|_{L^{4}(M)}^{1 / 2}\|u\|_{H^{1}(M)}^{1 / 2} .
\end{aligned}
$$

(ii) for $u \in V_{i}(i=1,2)$,

$$
\|u\|_{4} \leq C\|u\|_{2}^{1 / 4}\|u\|^{3 / 4}
$$

Proposition 2. There exists a positive constant $\lambda$ such that

$$
\|v\|^{2} \geq \lambda\|v\|_{2}^{2}
$$

for any $v \in V_{1}$.

Lemma 3 (see $[4,6]$ ). Let $v, v_{1} \in V_{1}, T \in V_{2}$, and $p \geq 1$. Then, we have

(1) $\int_{\Omega}\left[(v \cdot \nabla) v_{1}-\left(\int_{-h}^{z} e^{(z-\zeta) / H_{s}} \nabla \cdot v(x, y, \zeta) d \zeta\right)\left(\partial v_{1} / \partial z\right)\right] \cdot$ $\left(e^{-z / H_{s}}\left|v_{1}\right|^{p-1} v_{1}\right)=0$,

(2) $\int_{\Omega}\left[v \cdot \nabla T-\left(\int_{-h}^{z} e^{(z-\zeta) / H_{s}} \nabla \cdot v(x, y, \zeta) d \zeta\right)(\partial T /\right.$ $\partial z)] e^{-z / H_{s}}|T|^{p-1} T=0$,

(3) $\int_{\Omega} e^{-z / H_{s}} \nabla \Phi_{s}(x, y) \cdot v(x, y, z)=0$.

Lemma 4 (see [4], (Minkowski inequality)). Let $(X, \mu),(Y, v)$ be two measure spaces and $f(x, y)$ a measurable function about $\mu \times \nu$ on $X \times Y$, and $\rho(y)$ is a measurable positive function. If $f(\cdot, y) \in L^{p}(X, \mu)(1 \leq p \leq \infty)$ for a.e.y $\in Y$ and $\int_{Y} \rho(y)\|f(\cdot, y)\|_{L^{p}(X, \mu)} d v(y)<\infty$, then

$$
\begin{aligned}
& \left\|\int_{Y} \rho(y) f(\cdot, y) d v(y)\right\|_{L^{p}(X, \mu)} \\
& \quad \leq \int_{Y} \rho(y)\|f(\cdot, y)\|_{L^{p}(X, \mu)} d v(y) .
\end{aligned}
$$

Finally, we will recall the definition of strong solutions and state it as follows.

Definition 5. Assume that $\left(v_{0}, T_{0}\right) \in V$ and $Q \in H^{1}$ and let $\tau$ be any fixed positive time. $(v, T)$ is called a strong solution of (14)-(18) on $[0, \tau]$, if

$$
\begin{gathered}
(v, T) \in L^{\infty}(0, \tau ; V) \cap L^{2}\left(0, \tau ; H^{2}\right), \\
\left(v_{t}, T_{t}\right) \in L^{1}\left(0, \tau ; L^{2}\right),
\end{gathered}
$$


and they satisfy

$$
\begin{array}{r}
(v(t), \phi) \\
+\int_{t_{0}}^{t} \int_{\Omega} e^{-z / H_{s}}\left[(v \cdot \nabla) v-\left(\int_{-h}^{z} e^{(z-\zeta) / H_{s}} \nabla \cdot v(x, y, \zeta, t) d \zeta\right)\right. \\
\left.\quad \times \frac{\partial v}{\partial z}+f_{0} \vec{k} \times v+\frac{R}{H_{s}} \int_{0}^{z} \nabla T(x, y, \zeta, t) d \zeta\right] \\
\cdot \phi+\int_{t_{0}}^{t}((v, \phi)) \\
=\frac{1}{H_{s} R_{2}} \int_{t_{0}}^{t}\left(\partial_{z} v, \phi\right)+\left(v\left(t_{0}\right), \phi\right), \\
(T+t), \psi) \\
+\int_{t_{0}}^{t} \int_{\Omega} e^{-z / H_{s}}\left[v \cdot \nabla T-\left(\int_{-h}^{z} e^{(z-\zeta) / H_{s}} \nabla \cdot v(x, y, \zeta, t) d \zeta\right)\right. \\
=\int_{t_{0}}^{t}(Q, \psi)+\frac{\partial T}{H_{s} R t_{2}} \int_{t_{0}}^{t}\left(\partial_{z} T, \psi\right)+\left(T\left(t_{0}\right), \psi\right),
\end{array}
$$

for every $\phi \in \mathscr{V}_{1}$ and $\psi \in \mathscr{V}_{2}$, almost every $t_{0}, t \in[0, \tau]$.

\section{Some a Priori Estimates for (1)-(10)}

We start with the following general existence and uniqueness of solutions which can be obtained by the standard FatouGalerkin methods [20-22] and similar methods in [4]. Here, we only state it as follows.

Theorem 6. Assume that $\left(v_{0}, T_{0}\right) \in V$ and $Q \in H^{1}$ and let $\tau$ be any fixed positive time. Then, there exists a unique strong solution $(v, T)$ of $(14)-(18)$ on $[0, \tau]$, which satisfies

$$
(v, T) \in L^{\infty}(0, \tau ; V) \cap L^{2}\left(0, \tau ; H^{2}\right)
$$

and depends continuously on the initial data $\left(v_{0}, T_{0}\right)$; that is, the mapping $\left(v_{0}, T_{0}\right) \rightarrow(v, T)$ is continuous in $V$.

By Theorem 6, we can define the operator semigroup $\{S(t)\}_{t \geq 0}$ in $V$ as

$$
S(\cdot)\left(v_{0}, T_{0}\right): \mathbb{R}^{+} \times V \longrightarrow V,
$$

which is $(V, V)$-continuous.

In this section, we will give some a priori estimates which imply the existence of the $(X, Z)$-absorbing set $B_{0}$ for (1)-(10). That is, for any bounded subset $B \subset X$, there exists $T=T(B)$ such that $S(t) B \subset B_{0}$ for any $t \geq T$, where $\{S(t)\}_{t>0}$ is a semigroup on Banach space $X$ generated by (1)-(10).
3.1. $L^{2}$ Estimates of $T$. Taking the $L^{2}$ inner product of (15) with $T$, using the boundary conditions (17) and using Lemma 3, we deduce that

$$
\frac{1}{2} \frac{d}{d t}\|T\|_{2}^{2}+\|T\|^{2} \leq\|Q\|_{2}\|T\|_{2}+\frac{1}{H_{s} R t_{2}}\left\|\frac{\partial T}{\partial z}\right\|_{2}\|T\|_{2} .
$$

Employing Hölder's inequality yields

$$
\frac{d}{d t}\|T\|_{2}^{2}+\|T\|^{2} \leq \frac{2}{H_{s}^{2} R t_{2}}\|T\|_{2}^{2}+H_{s}^{2} R t_{2}\|Q\|_{2}^{2} .
$$

Thanks to

$$
\|T\|_{2}^{2} \leq 2 h\|T(z=0)\|_{L^{2}(M)}^{2}+h^{2}\left\|\frac{\partial T}{\partial z}\right\|_{2}^{2}+\frac{h^{2}}{4 H_{s}^{2}}\|T\|_{2}^{2},
$$

we obtain

$$
\begin{aligned}
& \frac{\|T\|_{2}^{2}}{h^{2} R t_{2}+2 h / \alpha} \\
& \quad \leq \frac{4 H_{s}^{2}}{4 H_{s}^{2}-h^{2}}\left(\frac{1}{R t_{2}}\left\|\frac{\partial T}{\partial z}\right\|_{2}^{2}+\alpha\|T(z=0)\|_{L^{2}(M)}^{2}\right) .
\end{aligned}
$$

Setting $\delta=1-8\left(R t_{2} h^{2}+2 h / \alpha\right) / R t_{2}\left(4 H_{s}^{2}-h^{2}\right)$ and $\delta_{1}=$ $\delta\left(4 H_{s}^{2}-h^{2}\right) / 4 H_{s}^{2}\left(R t_{2} h^{2}+2 h / \alpha\right)$, we find that

$$
\begin{aligned}
& \frac{d}{d t}\|T\|_{2}^{2}+\delta\|T\|^{2} \leq H_{s}^{2} R t_{2}\|Q\|_{2}^{2}, \\
& \frac{d}{d t}\|T\|_{2}^{2}+\delta_{1}\|T\|_{2}^{2} \leq H_{s}^{2} R t_{2}\|Q\|_{2}^{2},
\end{aligned}
$$

which implies that

$$
\|T(t)\|_{2}^{2} \leq\left\|T_{0}\right\|_{2}^{2} e^{-\delta_{1} t}+\frac{H_{s}^{2} R t_{2}}{\delta_{1}}\|Q\|_{2}^{2}\left(1-e^{-\delta_{1} t}\right) .
$$

Therefore, there exist three positive constants $\rho_{1}, \rho_{2}, T_{1}$ such that

$$
\begin{gathered}
\|T(t)\|_{2}^{2} \leq \rho_{1}, \\
\int_{t}^{t+1}\|T\|^{2} d \tau \leq \rho_{2},
\end{gathered}
$$

for any $t \geq T_{1}$. For brevity, we omit writing out explicitly these bounds here, and we also omit writing out other similar bounds in our future discussion for all other uniform a priori estimates.

3.2. $L^{2}$ Estimates of $v$. Multiplying (14) by $v$, integrating over $\Omega$, and using Lemma 3, we deduce that

$$
\frac{1}{2} \frac{d}{d t}\|v\|_{2}^{2}+\|v\|^{2} \leq C\|T\|_{2}\|\nabla v\|_{2}+\frac{1}{H_{s} \operatorname{Re}_{2}}\left\|\frac{\partial v}{\partial z}\right\|_{2}\|v\|_{2} .
$$


Setting $\delta_{3}=1-1 / \operatorname{Re}_{2} \lambda H_{s}^{2}$ and $\delta_{4}=\lambda-1 / \operatorname{Re}_{2} H_{s}^{2}$, we obtain

$$
\begin{aligned}
& \frac{d}{d t}\|v\|_{2}^{2}+\delta_{3}\|v\|^{2} \leq C\|T\|_{2}^{2}, \\
& \frac{d}{d t}\|v\|_{2}^{2}+\delta_{4}\|v\|_{2}^{2} \leq C\|T\|_{2}^{2} .
\end{aligned}
$$

From the classical Gronwall inequality, we have

$$
\|v(t)\|_{2}^{2} \leq\left\|v_{0}\right\|_{2}^{2} e^{-\delta_{4} t}+C \rho_{1}
$$

for all $t \geq T_{1}$, which implies that there exist three positive constants $\rho_{3}, \rho_{4}, T_{2} \geq T_{1}$ such that

$$
\begin{gathered}
\|v(t)\|_{2}^{2} \leq \rho_{3}, \\
\int_{t}^{t+1}\|v\|^{2} d \tau \leq \rho_{4},
\end{gathered}
$$

for all $t \geq T_{2}$.

3.3. $L^{6}(\Omega)$ Estimates of $T$. Taking the $L^{2}(\Omega)$ inner product of (15) with $|T|^{4} T$ and using Lemma 3 , we have

$$
\frac{1}{6} \frac{d}{d t}\|T\|_{6}^{6}+\frac{5}{9}\left\||T|^{3}\right\|^{2} \leq\left\||T|^{3}\right\|_{10 / 3}^{5 / 3}\|Q\|_{2}+\frac{1}{H_{s} R t_{2}}\left\||T|^{3}\right\|_{10 / 3}^{5 / 3}\left\|T_{z}\right\|_{2} .
$$

From the inequality $\|\varphi\|_{10 / 3} \leq C\|\varphi\|_{2}^{2 / 5}\|\varphi\|^{3 / 5}$, we get

$$
\frac{d}{d t}\|T\|_{6}^{6}+2\left\||T|^{3}\right\|^{2} \leq C\left\|T_{z}\right\|_{2}^{2}\|T\|_{6}^{4}+C\|Q\|_{2}^{2}\|T\|_{6}^{4},
$$

which implies that

$$
\frac{d}{d t}\|T\|_{6}^{2} \leq C\left\|T_{z}\right\|_{2}^{2}+C\|Q\|_{2}^{2}
$$

Therefore, by virtue of the uniform Gronwall lemma, from (44) and $\|T\|_{6} \leq C\|T\|_{H^{1}(\Omega)}$, we deduce that

$$
\|T\|_{6}^{2} \leq \rho_{5},
$$

for any $t \geq T_{2}+1$.
3.4. $\left(L^{6}(\Omega)\right)^{2}$ Estimates of $\widetilde{v}$. Multiplying (23) by $|\widetilde{v}|^{4} \widetilde{v}$, integrating over $\Omega$, and using Lemma 3 , we deduce that

$$
\begin{aligned}
& \frac{1}{6} \frac{d}{d t}\|\widetilde{v}\|_{6}^{6}+\frac{1}{\operatorname{Re}_{1}} \int_{\Omega} e^{-z / H_{s}}|\nabla \widetilde{v}|^{2}|\widetilde{v}|^{4} d x d y d z \\
& \quad+\frac{1}{\operatorname{Re}_{2}} \int_{\Omega} e^{-z / H_{s}}\left|\partial_{z} \widetilde{v}\right|^{2}|\widetilde{v}|^{4} d x d y d z+\frac{4}{9 \operatorname{Re}_{1}} \\
& \quad \times\left.\left.\int_{\Omega} e^{-z / H_{s}}|\nabla| \widetilde{v}\right|^{3}\right|^{2} d x d y d z \\
& \quad+\left.\left.\frac{4}{9 \operatorname{Re}_{2}} \int_{\Omega} e^{-z / H_{s}}\left|\partial_{z}\right| \widetilde{v}\right|^{3}\right|^{2} d x d y d z \\
& \leq \frac{1}{\operatorname{Re}_{2} H_{s}}\left\|\widetilde{v}_{z}\right\|_{2}\left\||\widetilde{v}|^{3}\right\|_{10 / 3}^{5 / 3}+C\|\bar{v}\|_{4}\|\widetilde{v}\|_{6}^{3 / 2}\left\||\widetilde{\mid}|^{3}\right\|^{1 / 2} \\
& \quad \times\left(\int_{\Omega}|\nabla \widetilde{v}|^{2}|\widetilde{v}|^{4} d x d y d z\right)^{1 / 2}+C\|\nabla T\|_{2}\|\widetilde{v}\|_{6}^{2}\left\||\widetilde{v}|^{3}\right\| \\
& \quad+\int_{\Omega} e^{-z / H_{s}} \frac{(\widetilde{v} \cdot \nabla) \widetilde{v}+(\nabla \cdot \widetilde{v}) \widetilde{v} \cdot|\widetilde{v}|^{4} \widetilde{v} d x d y d z}{}
\end{aligned}
$$

It follows from the Sobolev interpolation inequality and Lemma 4 that

$$
\begin{aligned}
& \int_{\Omega} e^{-z / H_{s}}\bar{v} \cdot \nabla) \widetilde{v}+(\nabla \cdot \widetilde{v}) \widetilde{v} \cdot|\widetilde{v}|^{4} \widetilde{v} d x d y d z \\
& \leq C \int_{M}\left(\int_{-h}^{0} e^{-z / H_{s}}|\nabla \widetilde{v}||\widetilde{v}|^{4} d z\right) \\
& \times\left(\int_{-h}^{0} e^{-z / H_{s}}|\widetilde{v}|^{2} d z\right) d x d y \\
& \leq C \int_{M}\left(\int_{-h}^{0} e^{-z / H_{s}}|\nabla \widetilde{v}|^{2}|\widetilde{v}|^{4} d z\right)^{1 / 2} \\
& \times\left(\int_{-h}^{0} e^{-z / H_{s}}|\widetilde{v}|^{4} d z\right) d x d y \\
& \leq C\left(\int_{\Omega} e^{-z / H_{s}}|\nabla \widetilde{v}|^{2}|\widetilde{v}|^{4} d x d y d z\right)^{1 / 2} \\
& \times\left(\int_{M}\left(\int_{-h}^{0} e^{-z / H_{s}}|\widetilde{v}|^{4} d z\right)^{2} d x d y\right)^{1 / 2}, \\
&\left(\int_{M}\left(\int_{-h}^{0} e^{-z / H_{s}}|\widetilde{v}|^{4} d z\right)^{2} d x d y\right)^{1 / 2} \\
& \leq \int_{-h}^{0} e^{-z / H_{s}}\left(\int_{M}|\widetilde{v}|^{8} d x d y\right)^{1 / 2} d z \\
& \leq C \int_{-h}^{0} e^{-z / H_{s}}\|\widetilde{v}\|_{L^{6}(M)}^{3}\|\widetilde{v}\|_{H^{1}(M)} d z \\
& \leq C\|\widetilde{v}\|_{6}^{3}\|\widetilde{v}\|,
\end{aligned}
$$


which implies that

$$
\begin{aligned}
& \int_{\Omega} e^{-z / H_{s}} \overline{(\widetilde{v} \cdot \nabla) \widetilde{v}+(\nabla \cdot \widetilde{v}) \widetilde{v}} \cdot|\widetilde{v}|^{4} \widetilde{v} d x d y d z \\
& \quad \leq C\left(\int_{\Omega} e^{-z / H_{s}}|\nabla \widetilde{v}|^{2}|\widetilde{v}|^{4} d x d y d z\right)^{1 / 2}\|\widetilde{v}\|_{6}^{3}\|\widetilde{v}\|,
\end{aligned}
$$

where we use the inequality $\|\widetilde{v}\|_{L^{8}(M)}^{8} \leq C\|\widetilde{v}\|_{L^{6}(M)}^{6}\|\widetilde{v}\|_{H^{1}(M)}^{2}$. We deduce from (54) and (56) that

$$
\frac{d}{d t}\|\widetilde{v}\|_{6}^{2} \leq C\left(\|\widetilde{v}\|^{2}+\|\bar{v}\|_{4}^{4}\right)\|\widetilde{v}\|_{6}^{2}+C\left\|\widetilde{v}_{z}\right\|_{2}^{2}+C\|\nabla T\|_{2}^{2} .
$$

Therefore, it follows from (44)-(49) and the uniform Gronwall lemma that

$$
\begin{gathered}
\|\widetilde{v}\|_{6}^{2} \leq \rho_{6}, \\
\int_{t}^{t+1} \int_{\Omega} e^{-z / H_{s}}|\nabla \widetilde{v}|^{2}|\widetilde{v}|^{4} d x d y d z d \tau \leq \rho_{7},
\end{gathered}
$$

for any $t \geq T_{2}+2$.

3.5. $\left(H^{1}(M)\right)^{2}$ Estimates of $\bar{v}$. Taking the $L^{2}(\Omega)$ inner product of (21) with $-\Delta \bar{v}$ and combining the boundary conditions (22), we deduce that

$$
\begin{aligned}
& \frac{1}{2} \frac{d}{d t}\|\nabla \bar{v}\|_{L^{2}(M)}^{2}+\frac{1}{\operatorname{Re}_{1}} \int_{M}|\Delta \bar{v}|^{2} d x d y \\
& \leq \frac{1}{\operatorname{Re}_{2} H_{s}}\left\|\widetilde{v}_{z}\right\|_{2}\|\Delta \bar{v}\|_{2}+C\|\bar{v}\|_{4}\|\nabla \bar{v}\|_{4}\|\Delta \bar{v}\|_{2} \\
& \quad+C \int_{M}\left(\int_{-h}^{0} e^{-z / H_{s}}|\nabla \widetilde{v}||\widetilde{v}| d z\right)|\Delta \bar{v}| d x d y \\
& \quad+C\|\bar{v}\|_{2}\|\Delta \bar{v}\|_{2}+C\|\nabla T\|_{2}\|\Delta \bar{v}\|_{2} .
\end{aligned}
$$

By using the Hölder inequality, we get

$$
\begin{aligned}
& \int_{M}\left(\int_{-h}^{0} e^{-z / H_{s}}|\nabla \widetilde{v}||\widetilde{v}| d z\right)|\Delta \bar{v}| d x d y \\
& \leq \int_{M}\left(\int_{-h}^{0} e^{-z / H_{s}}|\nabla \widetilde{v}| d z\right)^{1 / 2} \\
& \quad \times\left(\int_{-h}^{0} e^{-z / H_{s}}|\nabla \widetilde{v}||\widetilde{v}|^{2} d z\right)^{1 / 2}|\Delta \bar{v}| d x d y
\end{aligned}
$$

$$
\begin{aligned}
& \leq\left(\int_{M}\left(\int_{-h}^{0} e^{-z / H_{s}}|\nabla \widetilde{v}| d z\right)^{2} d x d y\right)^{1 / 4} \\
& \quad \times\left(\int_{M}\left(\int_{-h}^{0} e^{-z / H_{s}}|\nabla \widetilde{v}||\widetilde{v}|^{2} d z\right)^{2} d x d y\right)^{1 / 4}\|\Delta \bar{v}\|_{2} \\
& \leq\left(\int_{-h}^{0} e^{-z / H_{s}}\left(\int_{M}|\nabla \widetilde{v}|^{2} d x d y\right)^{1 / 2} d z\right)^{1 / 2} \\
& \quad \times\left(\int_{-h}^{0} e^{-z / H_{s}}\left(\int_{M}|\nabla \widetilde{v}|^{2}|\widetilde{v}|^{4} d x d y\right)^{1 / 2} d z\right)^{1 / 2}\|\Delta \bar{v}\|_{2} \\
& \leq C\|\nabla \widetilde{v}\|_{2}^{1 / 2}\left(\int_{\Omega} e^{-z / H_{s}}|\nabla \widetilde{v}|^{2}|\widetilde{v}|^{4} d x d y d z\right)^{1 / 4}\|\Delta \bar{v}\|_{2} .
\end{aligned}
$$

It follows from (60) and (61) that

$$
\begin{aligned}
& \frac{d}{d t}\|\nabla \bar{v}\|_{L^{2}(M)}^{2}+\frac{1}{\operatorname{Re}_{1}} \int_{M}|\Delta \bar{v}|^{2} d x d y \\
& \leq C\|\bar{v}\|_{L^{2}(M)}^{2}\|\nabla \bar{v}\|_{L^{2}(M)}^{4}+C\left\|\widetilde{v}_{z}\right\|_{2}^{2}+C\|\nabla T\|_{2}^{2}+C\|\nabla \widetilde{v}\|_{2}^{2} \\
& \quad+C\left(\int_{\Omega} e^{-z / H_{s}}|\nabla \widetilde{v}|^{2}|\widetilde{v}|^{4} d x d y d z\right)+C\|\bar{v}\|_{2}^{2} .
\end{aligned}
$$

In view of (44)-(49) and (59) and the uniform Gronwall lemma, we obtain

$$
\|\nabla \bar{v}\|_{L^{2}(M)}^{2} \leq \rho_{8}
$$

for any $t \geq T_{2}+3$.

3.6. $\left(L^{2}(\Omega)\right)^{2}$ Estimates of $v_{z}$. Denote that $u=v_{z}$. It is clear that $u$ satisfies the following equation obtained by differentiating (14) with respect to $z$ :

$$
\begin{aligned}
\frac{\partial u}{\partial t}+ & L_{1} u+(v \cdot \nabla) u \\
& -\left(\int_{-h}^{z} e^{(z-\zeta) / H_{s}} \nabla \cdot v(x, y, \zeta, t) d \zeta\right) \frac{\partial u}{\partial z} \\
& -\frac{1}{H_{s}}\left(\int_{-h}^{z} e^{(z-\zeta) / H_{s}} \nabla \cdot v(x, y, \zeta, t) d \zeta\right) u \\
& +(u \cdot \nabla) v-(\nabla \cdot v) u+f_{0} \vec{k} \times u+\frac{R}{H_{s}} \nabla T=0,
\end{aligned}
$$

with the boundary conditions

$$
\begin{aligned}
& \left.u\right|_{\Gamma_{u}}=0,\left.\quad u\right|_{\Gamma_{b}}=0, \\
& \left.u \cdot \vec{n}\right|_{\Gamma_{l}}=0, \quad \frac{\partial u}{\partial \vec{n}} \times\left.\vec{n}\right|_{\Gamma_{l}}=0 .
\end{aligned}
$$


Multiplying (64) by $u$, integrating over $\Omega$, and using Lemma 3, we get

$$
\begin{aligned}
& \frac{1}{2} \frac{d}{d t}\|u\|_{2}^{2}+\|u\|^{2} \\
& \quad \leq \frac{1}{\operatorname{Re}_{2} H_{s}}\left\|u_{z}\right\|_{2}\|u\|_{2}+C\|v\|_{6}\|u\|_{3}\|\nabla u\|_{2}+C\|T\|_{2}\|\nabla u\|_{2} .
\end{aligned}
$$

It follows from the Young inequality and the Sobolev embedding theorem that

$$
\frac{d}{d t}\|u\|_{2}^{2}+\|u\|^{2} \leq C\left(1+\|v\|_{6}^{4}\right)\|u\|_{2}^{2}+C\|T\|_{2}^{2} .
$$

Since

$$
\|v\|_{6} \leq C h^{-1 / 3}\|v\|_{2}+C h^{1 / 6}\|\nabla \bar{v}\|_{2}+\|\widetilde{v}\|_{6},
$$

which implies that

$$
\|v\|_{6}^{2} \leq \rho_{9}
$$

for any $t \geq T_{2}+3$.

By virtue of the uniform Gronwall lemma, from (43), (49), and (69), we deduce that

$$
\begin{gathered}
\left\|v_{z}\right\|_{2}^{2} \leq \rho_{10}, \\
\int_{t}^{t+1}\left\|v_{z}\right\|^{2} d \tau \leq \rho_{11},
\end{gathered}
$$

for any $t \geq T_{2}+4$.

3.7. $\left(L^{2}(\Omega)\right)^{2}$ Estimates of $\nabla v$. Taking the $L^{2}(\Omega)$ inner product of (14) with $-\Delta v$ and combining the boundary conditions (16), we deduce that

$$
\begin{aligned}
\frac{1}{2} \frac{d}{d t}\|\nabla v\|_{2}^{2}+\frac{1}{\operatorname{Re}_{1}} \int_{\Omega} e^{-z / H_{s}}|\Delta v|^{2} d x d y d z \\
\quad+\frac{1}{\operatorname{Re}_{2}} \int_{\Omega} e^{-z / H_{s}}\left|\nabla v_{z}\right|^{2} d x d y d z \\
\leq \frac{1}{\operatorname{Re}_{2} H_{s}}\left\|v_{z}\right\|_{2}\|\Delta v\|_{2}+C\left\|v_{z}\right\|_{2}^{1 / 2}\left\|\nabla v_{z}\right\|_{2}^{1 / 2}\|\nabla v\|_{2}^{1 / 2}\|\Delta v\|_{2}^{3 / 2} \\
\quad+C\|\nabla T\|_{2}\|\Delta v\|_{2}+C\|v\|_{6}\|\nabla v\|_{3}\|\Delta v\|_{2}+C\|v\|_{2}\|\Delta v\|_{2} .
\end{aligned}
$$

We derive from (72) and the Young inequality that

$$
\begin{aligned}
& \frac{d}{d t}\|\nabla v\|_{2}^{2}+\frac{1}{\operatorname{Re}_{1}} \int_{\Omega} e^{-z / H_{s}}|\Delta v|^{2} d x d y d z \\
& \quad+\frac{1}{\operatorname{Re}_{2}} \int_{\Omega} e^{-z / H_{s}}\left|\nabla v_{z}\right|^{2} d x d y d z \\
& \leq C\left(\|v\|_{6}^{4}+\left\|v_{z}\right\|_{2}^{2}\left\|\nabla v_{z}\right\|_{2}^{2}\right)\|\nabla v\|_{2}^{2}+C\|\nabla T\|_{2}^{2}+C\|v\|_{2}^{2}+C\left\|v_{z}\right\|_{2}^{2} .
\end{aligned}
$$

By virtue of (44)-(49), (69)-(71), and the uniform Gronwall lemma, we get

$$
\begin{gathered}
\|\nabla v\|_{2}^{2} \leq \rho_{12}, \\
\frac{1}{\operatorname{Re}_{1}} \int_{t}^{t+1} \int_{\Omega} e^{-z / H_{s}}|\Delta v|^{2} d x d y d z d \tau \\
+\frac{1}{\operatorname{Re}_{2}} \int_{t}^{t+1} \int_{\Omega} e^{-z / H_{s}}\left|\nabla v_{z}\right|^{2} d x d y d z d \tau \leq \rho_{13},
\end{gathered}
$$

for any $t \geq T_{2}+5$.

3.8. $L^{2}(\Omega)$ Estimates of $T_{z}$. Denote $\theta=T_{z}$. It is clear that $\theta$ satisfies the following equation obtained by differentiating (15) with respect to $z$ :

$$
\begin{aligned}
\frac{\partial \theta}{\partial t} & +L_{2} \theta+v \cdot \nabla \theta-\left(\int_{-h}^{z} e^{(z-\zeta) / H_{s}} \nabla \cdot v(x, y, \zeta, t) d \zeta\right) \frac{\partial \theta}{\partial z} \\
& -\frac{1}{H_{s}}\left(\int_{-h}^{z} e^{(z-\zeta) / H_{s}} \nabla \cdot v(x, y, \zeta, t) d \zeta\right) \theta \\
& +v_{z} \cdot \nabla T-(\nabla \cdot v) \theta=\partial_{z} Q
\end{aligned}
$$

with the boundary conditions

$$
\left.\left(\frac{1}{R t_{2}} \theta+\alpha T\right)\right|_{\Gamma_{u}}=0,\left.\quad \theta\right|_{\Gamma_{b}}=0,\left.\quad \frac{\partial \theta}{\partial \vec{n}}\right|_{\Gamma_{l}}=0 .
$$

Multiplying (76) by $\theta$, integrating over $\Omega$, and using Lemma 3, we deduce that

$$
\begin{aligned}
\frac{1}{2} \frac{d}{d t}\|\theta\|_{2}^{2}+\frac{1}{R t_{1}} \int_{\Omega} e^{-z / H_{s}}|\nabla \theta|^{2} d x d y d z \\
\quad+\frac{1}{R t_{2}} \int_{\Omega} e^{-z / H_{s}}\left|\partial_{z} \theta\right|^{2} d x d y d z+\alpha \int_{\Gamma_{u}} \frac{\partial \theta}{\partial z} T d x d y \\
\leq \frac{1}{R t_{2} H_{s}}\left\|\theta_{z}\right\|_{2}\|\theta\|_{2}+C\|v\|_{6}\|\theta\|_{3}\|\nabla \theta\|_{2}+C\left\|Q_{z}\right\|_{2}\|\theta\|_{2} \\
\quad+C\left\|\nabla v_{z}\right\|_{2}\|T\|_{6}\|\theta\|_{3}+C\left\|v_{z}\right\|_{3}\|T\|_{6}\|\nabla \theta\|_{2} .
\end{aligned}
$$


We deduce from the Young inequality that

$$
\begin{aligned}
\alpha \int_{\Gamma_{u}} \frac{\partial \theta}{\partial z} T d x d y & \\
= & \alpha R t_{2} \int_{\Gamma_{u}}\left(\frac{\partial T}{\partial t}+v \cdot \nabla T-\frac{1}{R t_{1}} \Delta T-Q\right) T d x d y \\
= & \frac{\alpha R t_{2}}{2} \frac{d}{d t} \int_{\Gamma_{u}}|T|^{2} d x d y+\alpha R t_{2} \int_{\Gamma_{u}}(v \cdot \nabla T) T d x d y \\
& +\frac{\alpha R t_{2}}{R t_{1}} \int_{\Gamma_{u}}|\nabla T|^{2} d x d y-\alpha R t_{2} \int_{\Gamma_{u}} Q T d x d y, \\
\leq R & \int_{\Gamma_{u}}(v \cdot \nabla T) T d x d y-\alpha R t_{2} \int_{\Gamma_{u}} Q T d x d y \mid \\
\leq & C\|v\|_{L^{4}\left(\Gamma_{u}\right)}\|\nabla T\|_{L^{2}\left(\Gamma_{u}\right)}\|T\|_{L^{4}\left(\Gamma_{u}\right)}+C\|Q\|_{L^{2}\left(\Gamma_{u}\right)}\|T\|_{L^{2}\left(\Gamma_{u}\right)} \\
\leq & C\|v\|\|\nabla T\|_{L^{2}\left(\Gamma_{u}\right)}^{3 / 2}\|T\|_{L^{2}\left(\Gamma_{u}\right)}^{1 / 2}+C\|Q\|\|T\|_{L^{2}\left(\Gamma_{u}\right)} .
\end{aligned}
$$

It follows from (78) and (79) that

$$
\begin{aligned}
& \frac{d}{d t}\left(\|\theta\|_{2}^{2}+\alpha R t_{2}\|T\|_{L^{2}\left(\Gamma_{u}\right)}^{2}\right) \\
& \quad+\frac{1}{R t_{1}} \int_{\Omega} e^{-z / H_{s}}|\nabla \theta|^{2} d x d y d z \\
& \quad+\frac{1}{R t_{2}} \int_{\Omega} e^{-z / H_{s}}\left|\theta_{z}\right|^{2} d x d y d z+\frac{\alpha R t_{2}}{R t_{1}} \int_{\Gamma_{u}}|\nabla T|^{2} d x d y \\
& \leq C\left(1+\|v\|_{6}^{4}+\|T\|_{6}^{4}+\|v\|^{4}\right)\left(\|\theta\|_{2}^{2}+\alpha R t_{2}\|T\|_{L^{2}\left(\Gamma_{u}\right)}^{2}\right) \\
& \quad+C\left(\|Q\|^{2}+\left\|v_{z}\right\|^{2}+\left\|v_{z}\right\|_{2}^{2}\|T\|_{6}^{4}\right) .
\end{aligned}
$$

As a result of (44), (53), (69)-(71), and (74), we get

$$
\begin{gathered}
\left\|T_{z}\right\|_{2}^{2}+\alpha R t_{2}\|T\|_{L^{2}\left(\Gamma_{u}\right)}^{2} \leq \rho_{14}, \\
\frac{1}{R t_{1}} \int_{t}^{t+1} \int_{\Omega} e^{-z / H_{s}}\left|\nabla T_{z}\right|^{2} d x d y d z d \tau \\
+\frac{1}{R t_{2}} \int_{t}^{t+1} \int_{\Omega} e^{-z / H_{s}}\left|\partial_{z}^{2} T\right|^{2} d x d y d z d \tau \\
+\frac{\alpha R t_{2}}{R t_{1}} \int_{t}^{t+1} \int_{\Gamma_{u}}|\nabla T|^{2} d x d y d \tau \leq \rho_{15},
\end{gathered}
$$

for any $t \geq T_{2}+6$.
3.9. $L^{2}(\Omega)$ Estimates of $\nabla T$. Taking the $L^{2}(\Omega)$ inner product of (15) with $-\Delta T$ and combining the boundary conditions (17), we deduce that

$$
\begin{aligned}
\frac{1}{2} \frac{d}{d t}\|\nabla T\|_{2}^{2}+\frac{1}{R t_{1}} \int_{\Omega} e^{-z / H_{s}}|\Delta T|^{2} d x d y d z \\
\quad+\frac{1}{R t_{2}} \int_{\Omega}\left|\nabla T_{z}\right|^{2} d x d y d z+\alpha \int_{\Gamma_{u}}|\nabla T|^{2} d x d y \\
\leq \frac{1}{R t_{2} H_{s}}\left\|T_{z}\right\|_{2}\|\Delta T\|_{2}+C\|v\|_{6}\|\nabla T\|_{3}\|\Delta T\|_{2}+C\|Q\|_{2}\|\Delta T\|_{2} \\
\quad+C\|\nabla v\|_{2}^{1 / 2}\|\Delta v\|_{2}^{1 / 2}\left\|T_{z}\right\|_{2}^{1 / 2}\left\|\nabla T_{z}\right\|_{2}^{1 / 2}\|\Delta T\|_{2} .
\end{aligned}
$$

We derive from the Young inequality that

$$
\begin{aligned}
& \frac{d}{d t}\|\nabla T\|_{2}^{2}+\frac{1}{R t_{1}} \int_{\Omega} e^{-z / H_{s}}|\Delta T|^{2} d x d y d z \\
& \quad+\frac{1}{R t_{2}} \int_{\Omega} e^{-z / H_{s}}\left|\nabla T_{z}\right|^{2} d x d y d z \\
& \quad+\alpha \int_{M}|\nabla T(z=0)|^{2} d x d y \\
& \leq C\|v\|_{6}^{4}\|\nabla T\|_{2}^{2}+C\|\nabla v\|_{2}^{2}\|\Delta v\|_{2}^{2}\left\|T_{z}\right\|_{2}^{2}+C\|Q\|_{2}^{2}+C\left\|T_{z}\right\|_{2}^{2} \text {. } \\
& \text { Using (44), (69), (74), (75), and (81), we get }
\end{aligned}
$$

$$
\|\nabla T\|_{2}^{2} \leq \rho_{16},
$$

$$
\begin{aligned}
& \frac{1}{R t_{1}} \int_{t}^{t+1} \int_{\Omega} e^{-z / H_{s}}|\Delta T|^{2} d x d y d z d \tau \\
& \quad+\frac{1}{R t_{2}} \int_{t}^{t+1} \int_{\Omega} e^{-z / H_{s}}\left|\nabla T_{z}\right|^{2} d x d y d z d \tau \\
& \quad+\alpha \int_{t}^{t+1} \int_{M}|\nabla T(z=0)|^{2} d x d y d \tau \\
& \quad \leq \rho_{17},
\end{aligned}
$$


3.10. $\left(L^{6}(\Omega)\right)^{2}$ Estimates of $\partial_{z} v$. Taking the $L^{2}(\Omega)$ inner product of (64) with $|u|^{4} u$, combining the boundary conditions (65), and using Lemma 3, we deduce that

$$
\begin{aligned}
\frac{1}{6} \frac{d}{d t}\|u\|_{6}^{6}+\frac{1}{\operatorname{Re}_{1}} \int_{\Omega} e^{-z / H_{s}}|\nabla u|^{2}|u|^{4} d x d y d z \\
\quad+\frac{1}{\operatorname{Re}_{2}} \int_{\Omega} e^{-z / H_{s}}\left|\partial_{z} u\right|^{2}|u|^{4} d x d y d z \\
\quad+\left.\left.\frac{4}{9 \operatorname{Re}_{1}} \int_{\Omega} e^{-z / H_{s}}|\nabla| u\right|^{3}\right|^{2} d x d y d z \\
\quad+\left.\left.\frac{4}{9 \operatorname{Re}_{2}} \int_{\Omega} e^{-z / H_{s}}\left|\partial_{z}\right| u\right|^{3}\right|^{2} d x d y d z \\
\leq \frac{1}{\operatorname{Re}_{2} H_{s}}\left\|u_{z}\right\|\left\|_{2}\right\||u|^{3}\left\|_{10 / 3}^{5 / 3}+C\right\| v\left\|_{6}\right\||u|^{3}\left\|_{3}\right\| \nabla|u|^{3} \|_{2} \\
+C\|\nabla T\|_{2}\left\||u|^{3}\right\|_{10 / 3}^{5 / 3}+C\|v\|_{6}\left\||u|^{3}\right\|_{3}\left\||\nabla u||u|^{2}\right\|_{2}
\end{aligned}
$$

From the Young inequality, we obtain

$$
\frac{d}{d t}\|u\|_{6}^{2} \leq C\|v\|_{6}^{4}\|u\|_{6}^{2}+C\|\nabla T\|_{2}^{2}+C\left\|u_{z}\right\|_{2}^{2}
$$

Thanks to (44), (69), and (71), we have

$$
\left\|v_{z}\right\|_{6}^{2} \leq \rho_{18}
$$

for any $t \geq T_{2}+8$.

3.11. $L^{6}(\Omega)$ Estimates of $\partial_{z} T$. Taking the $L^{2}(\Omega)$ inner product of (76) with $|\theta|^{4} \theta$, combining the boundary conditions (77), and using Lemma 3, we deduce that

$$
\begin{aligned}
& \frac{1}{6} \frac{d}{d t}\|\theta\|_{6}^{6}+\left.\left.\frac{5}{9 R t_{1}} \int_{\Omega} e^{-z / H_{s}}|\nabla| \theta\right|^{3}\right|^{2} d x d y d z \\
& \quad+\left.\left.\frac{5}{9 R t_{2}} \int_{\Omega} e^{-z / H_{s}}\left|\partial_{z}\right| \theta\right|^{3}\right|^{2} d x d y d z \\
& +\alpha^{5} R t_{2}^{4} \int_{\Gamma_{u}} \frac{\partial \theta}{\partial z}|T|^{4} T d x d y \\
& \leq \frac{1}{R t_{2} H_{s}}\left\|\theta_{z}\right\|_{2}\left\||\theta|^{3}\right\|_{10 / 3}^{5 / 3}+C\|v\|_{6}\left\||\theta|^{3}\right\|_{3}\left\|\nabla|\theta|^{3}\right\|_{2} \\
& \quad+C\|v\|_{6}\left\||\theta|^{3}\right\|_{3}\left\||\nabla \theta||\theta|^{2}\right\|_{2}+\left\|Q_{z}\right\|_{2}\left\||\theta|^{3}\right\|_{10 / 3}^{5 / 3} \\
& +\left.\left|-\int_{\Omega} e^{-z / H_{s}}\left(v_{z} \cdot \nabla T\right)\right| \theta\right|^{4} \theta d x d y d z \mid .
\end{aligned}
$$

It follows from the Hölder inequality that

$$
\begin{aligned}
& \left.\left|-\int_{\Omega} e^{-z / H_{s}}\left(v_{z} \cdot \nabla T\right)\right| \theta\right|^{4} \theta d x d y d z \mid \\
& \leq C \int_{\Omega} e^{-z / H_{s}}\left|v_{z}\right||\nabla T||\theta|^{5} d x d y d z \\
& \leq C\left\|v_{z}\right\|_{6}\|\nabla T\|_{3}\left\||\theta|^{3}\right\|_{10 / 3}^{5 / 3} \\
& \leq C\left\|v_{z}\right\|_{6}\|\nabla T\|_{2}^{1 / 2}\|\nabla T\|_{H^{1}(\Omega)}^{1 / 2}\left\||\theta|^{3}\right\|_{2}^{2 / 3}\left\||\theta|^{3}\right\|_{H^{1}(\Omega)}, \\
& \alpha^{5} R t_{2}^{4} \int_{\Gamma_{u}} \frac{\partial \theta}{\partial z}|T|^{4} T d x d y \\
& =\alpha^{5} R t_{2}^{5} \int_{\Gamma_{u}}\left(\frac{\partial T}{\partial t}+v \cdot \nabla T-\frac{1}{R t_{1}} \Delta T-Q\right)|T|^{4} T d x d y \\
& =\frac{\alpha^{5} R t_{2}^{5}}{6} \frac{d}{d t} \int_{\Gamma_{u}}|T|^{6} d x d y+\alpha^{5} R t_{2}^{5} \int_{\Gamma_{u}}(v \cdot \nabla T)|T|^{4} T d x d y \\
& +\left.\left.\frac{5 \alpha^{5} R t_{2}^{5}}{9 R t_{1}} \int_{\Gamma_{u}}|\nabla| T\right|^{3}\right|^{2} d x d y-\alpha^{5} R t_{2}^{5} \int_{\Gamma_{u}} Q|T|^{4} T d x d y, \\
& \left.\left|\alpha^{5} R t_{2}^{5} \int_{\Gamma_{u}}(v \cdot \nabla T)\right| T\right|^{4} T d x d y-\alpha^{5} R t_{2}^{5} \int_{\Gamma_{u}} Q|T|^{4} T d x d y \mid \\
& \leq C\|v\|_{L^{4}\left(\Gamma_{u}\right)}\left\|\nabla|T|^{3}\right\|_{L^{2}\left(\Gamma_{u}\right)}\left\||T|^{3}\right\|_{L^{4}\left(\Gamma_{u}\right)}+C\|Q\|_{L^{2}\left(\Gamma_{u}\right)}\left\||T|^{3}\right\|_{L^{10 / 3}\left(\Gamma_{u}\right)}^{5 / 3} \\
& \leq C\|v\|\left\|\nabla|T|^{3}\right\|_{L^{2}\left(\Gamma_{u}\right)}^{3 / 2}\|T\|_{L^{6}\left(\Gamma_{u}\right)}^{3 / 2}+C\|Q\|\left\||T|^{3}\right\|_{L^{2}\left(\Gamma_{u}\right)}\left\||T|^{3}\right\|_{H^{1}\left(\Gamma_{u}\right)}^{2 / 3} .
\end{aligned}
$$

We derive from the Young inequality that

$$
\begin{aligned}
& \frac{d}{d t}\left(\|\theta\|_{6}^{2}+\alpha R t_{2}\|T\|_{L^{6}\left(\Gamma_{u}\right)}^{2}\right) \\
& \leq C\left(1+\|v\|_{6}^{4}+\|v\|^{4}\right)\left(\|\theta\|_{6}^{2}+\alpha R t_{2}\|T\|_{L^{6}\left(\Gamma_{u}\right)}^{2}\right) \\
& \quad+C\left\|\theta_{z}\right\|_{2}^{2}+C\|Q\|^{2}+C\left\|v_{z}\right\|_{6}^{4}+C\|\nabla T\|_{2}^{2}\|\nabla T\|_{H^{1}(\Omega)}^{2}
\end{aligned}
$$

As a consequence of the uniform Gronwall lemma, we get

$$
\left\|T_{z}\right\|_{6}^{2}+\alpha R t_{2}\|T\|_{L^{6}\left(\Gamma_{u}\right)}^{2} \leq \rho_{19}
$$

for any $t \geq T_{2}+9$. 
3.12. H Estimates of $\left(\partial_{t} v, \partial_{t} T\right)$. Denote that $\pi=\partial_{t} v, \xi=\partial_{t} T$. It is clear that $\pi$, $\xi$ satisfy the following equations obtained by differentiating (14) and (15) with respect to $t$, respectively,

$$
\begin{aligned}
\frac{\partial \pi}{\partial t}+L_{1} \pi & +(v \cdot \nabla) \pi \\
& -\left(\int_{-h}^{z} e^{(z-\zeta) / H_{s}} \nabla \cdot v(x, y, \zeta, t) d \zeta\right) \frac{\partial \pi}{\partial z} \\
& +f_{0} \vec{k} \times \pi+\nabla \partial_{t} \Phi_{s}(x, y, t)+(\pi \cdot \nabla) v \\
& -\left(\int_{-h}^{z} e^{(z-\zeta) / H_{s}} \nabla \cdot \pi(x, y, \zeta, t) d \zeta\right) \frac{\partial v}{\partial z} \\
& +\frac{R}{H_{s}} \int_{0}^{z} \nabla \xi(x, y, \zeta, t) d \zeta=0 \\
\frac{\partial \xi}{\partial t}+L_{2} \xi & +v \cdot \nabla \xi-\left(\int_{-h}^{z} e^{(z-\zeta) / H_{s}} \nabla \cdot v(x, y, \zeta, t) d \zeta\right) \frac{\partial \xi}{\partial z} \\
+ & \pi \cdot \nabla T-\left(\int_{-h}^{z} e^{(z-\zeta) / H_{s}} \nabla \cdot \pi(x, y, \zeta, t) d \zeta\right) \frac{\partial T}{\partial z}=0
\end{aligned}
$$

with the boundary conditions

$$
\begin{array}{cc}
\left.\frac{\partial \pi}{\partial z}\right|_{\Gamma_{u}}=0, & \left.\frac{\partial \pi}{\partial z}\right|_{\Gamma_{b}}=0, \\
\left.\pi \cdot \vec{n}\right|_{\Gamma_{l}}=0, & \frac{\partial \pi}{\partial \vec{n}} \times\left.\vec{n}\right|_{\Gamma_{l}}=0, \\
\left.\left(\frac{1}{R t_{2}} \frac{\partial \xi}{\partial z}+\alpha \xi\right)\right|_{\Gamma_{u}}=0, & \left.\frac{\partial \xi}{\partial z}\right|_{\Gamma_{b}}=0,\left.\quad \frac{\partial \xi}{\partial \vec{n}}\right|_{\Gamma_{l}}=0,
\end{array}
$$

Multiplying (94) and (95), respectively, by $\pi$, $\xi$, integrating over $\Omega$, and using Lemma 3 , we deduce that

$$
\begin{aligned}
& \frac{1}{2} \frac{d}{d t}\|\pi\|_{2}^{2}+\|\pi\|^{2} \\
& \leq \frac{1}{\operatorname{Re}_{2} H_{s}}\left\|\pi_{z}\right\|_{2}\|\pi\|_{2}+C\|v\|_{6}\|\pi\|_{3}\|\nabla \pi\|_{2} \\
& \quad+C\left\|v_{z}\right\|\left\|_{6}\right\| \pi\left\|_{3}\right\| \nabla \pi\left\|_{2}+C\right\| \nabla \pi\left\|_{2}\right\| \xi \|_{2}, \\
& \frac{1}{2} \frac{d}{d t}\|\xi\|_{2}^{2}+\|\xi\|^{2} \\
& \leq \frac{1}{R t_{2} H_{s}}\left\|\xi_{z}\right\|_{2}\|\xi\|_{2}+C\|T\|_{6}\|\pi\|_{3}\|\nabla \xi\|_{2} \\
& \quad+C\|T\|_{6}\|\xi\|_{3}\|\nabla \pi\|_{2}+C\left\|T_{z}\right\|_{6}\|\xi\|_{3}\|\nabla \pi\|_{2} .
\end{aligned}
$$

It follows from the Young inequality that

$$
\begin{aligned}
& \frac{d}{d t}\left(\|\xi\|_{2}^{2}+\|\pi\|_{2}^{2}\right) \\
& \quad \leq C\left(1+\|T\|_{6}^{4}+\|v\|_{6}^{4}+\left\|T_{z}\right\|_{6}^{4}+\left\|v_{z}\right\|_{6}^{4}\right)\left(\|\xi\|_{2}^{2}+\|\pi\|_{2}^{2}\right) .
\end{aligned}
$$

Multiplying (15) by $\xi$ and integrating over $\Omega$, we obtain

$$
\begin{aligned}
\|\xi\|_{2}^{2} \leq & \|Q\|_{2}\|\xi\|_{2}+C\|v\|_{6}\|\nabla T\|_{3}\|\xi\|_{2} \\
& +\left\|L_{2} T\right\|_{2}\|\xi\|_{2}+C\left\|T_{z}\right\|_{6}\|\nabla v\|_{3}\|\xi\|_{2} .
\end{aligned}
$$

Similarly, we have

$$
\begin{aligned}
\|\pi\|_{2}^{2} \leq & C\|v\|_{6}\|\nabla v\|_{3}\|\pi\|_{2}+C\left\|v_{z}\right\|_{6}\|\nabla v\|_{3}\|\pi\|_{2} \\
& +C\|v\|_{2}\|\pi\|_{2}+C\|\nabla T\|_{2}\|\pi\|_{2}+\left\|L_{1} v\right\|_{2}\|\pi\|_{2} .
\end{aligned}
$$

We derive from the Young inequality that

$$
\begin{aligned}
&\|\pi\|_{2}^{2}+\|\xi\|_{2}^{2} \leq C\left(1+\|v\|_{6}^{4}+\left\|v_{z}\right\|_{6}^{4}+\left\|T_{z}\right\|_{6}^{4}\right)\left(\|\nabla T\|_{2}^{2}+\|\nabla v\|_{2}^{2}\right) \\
&+C\|Q\|_{2}^{2}+C\left\|L_{2} T\right\|_{2}^{2}+C\left\|L_{1} v\right\|_{2}^{2}+C\|v\|_{2}^{2} .
\end{aligned}
$$

By use of the uniform Gronwall inequality, we have

$$
\left\|v_{t}\right\|_{2}^{2}+\left\|T_{t}\right\|_{2}^{2} \leq \rho_{20},
$$

for any $t \geq T_{2}+10$.

Moreover, we have

$$
\int_{t}^{t+1}\left\|v_{t}\right\|^{2} d \tau+\int_{t}^{t+1}\left\|T_{t}\right\|^{2} d \tau \leq \rho_{21}
$$

for any $t \geq T_{2}+10$.

3.13. H Estimates of $\left(L_{1} v, L_{2} T\right)$. Multiplying (15) by $L_{2} T$ and integrating over $\Omega$, we obtain

$$
\begin{aligned}
\left\|L_{2} T\right\|_{2}^{2} \leq & C\|v\|_{6}\|\nabla T\|_{3}\left\|L_{2} T\right\|_{2}+C\left\|T_{z}\right\|_{6}\|\nabla v\|_{3}\left\|L_{2} T\right\|_{2} \\
& +\|Q\|_{2}\left\|L_{2} T\right\|_{2}+\left\|\partial_{t} T\right\|_{2}\left\|L_{2} T\right\|_{2} .
\end{aligned}
$$

Similarly, we have

$$
\begin{aligned}
\left\|L_{1} v\right\|_{2}^{2} \leq & C\|v\|_{6}\|\nabla v\|_{3}\left\|L_{1} v\right\|_{2}+C\left\|v_{z}\right\|_{6}\|\nabla v\|_{3}\left\|L_{1} v\right\|_{2} \\
& +C\|v\|_{2}\left\|L_{1} v\right\|_{2}+C\|\nabla T\|_{2}\left\|L_{1} v\right\|_{2}+\left\|v_{t}\right\|_{2}\left\|L_{1} v\right\|_{2} .
\end{aligned}
$$

It follows from the Young inequality that

$$
\begin{aligned}
\left\|L_{1} v\right\|_{2}^{2} & +\left\|L_{2} T\right\|_{2}^{2} \\
\leq & C\left(1+\|v\|_{6}^{4}+\left\|T_{z}\right\|_{6}^{4}+\left\|v_{z}\right\|_{6}^{4}\right)\left(\|\nabla T\|_{2}^{2}+\|\nabla v\|_{2}^{2}\right) \\
& +C\left(\left\|v_{t}\right\|_{2}^{2}+\left\|T_{t}\right\|_{2}^{2}\right)+C\left(\|v\|_{2}^{2}+\|Q\|_{2}^{2}\right) .
\end{aligned}
$$

By virtue of (48), (69), (74), (85), (89), (93), and (103), we have

$$
\left\|L_{1} v\right\|_{2}^{2}+\left\|L_{2} T\right\|_{2}^{2} \leq \rho_{22}
$$

for any $t \geq T_{2}+10$. 
3.14. $L^{2}$ Estimates of $\left(\nabla v_{t}, \nabla T_{t}\right)$. Taking the $H_{1}$ inner product of (94) with $-\Delta \pi$ and combining the boundary conditions (96), we have

$$
\begin{aligned}
& \frac{1}{2} \frac{d}{d t}\|\nabla \pi\|_{2}^{2}+\frac{1}{\operatorname{Re}_{1}} \int_{\Omega} e^{-z / H_{s}}|\Delta \pi|^{2} d x d y d z \\
& \quad+\frac{1}{\operatorname{Re}_{2}} \int_{\Omega} e^{-z / H_{s}}\left|\nabla \pi_{z}\right|^{2} d x d y d z \\
& \leq \frac{1}{\operatorname{Re}_{2} H_{s}}\left\|\pi_{z}\right\|_{2}\|\Delta \pi\|_{2}+C\|v\|_{6}\|\nabla \pi\|_{3}\|\Delta \pi\|_{2} \\
& \quad+C\|\nabla v\|_{2}^{1 / 2}\|\Delta v\|_{2}^{1 / 2}\left\|\pi_{z}\right\|_{2}^{1 / 2}\left\|\nabla \pi_{z}\right\|_{2}^{1 / 2}\|\Delta \pi\|_{2} \\
& \quad+C\|\pi\|_{2}\|\Delta \pi\|_{2}+C\|\pi\|_{6}\|\nabla v\|_{3}\|\Delta \pi\|_{2} \\
& \quad+C\|\nabla \pi\|_{2}^{1 / 2}\left\|v_{z}\right\|_{2}^{1 / 2}\left\|\nabla v_{z}\right\|_{2}^{1 / 2}\|\Delta \pi\|_{2}^{3 / 2}+C\|\nabla \xi\|_{2}\|\Delta \pi\|_{2} .
\end{aligned}
$$

Therefore, in view of the Young inequality, we obtain

$$
\begin{aligned}
\frac{d}{d t}\|\nabla \pi\|_{2}^{2}+\frac{1}{\operatorname{Re}_{1}} \int_{\Omega} e^{-z / H_{s}}|\Delta \pi|^{2} d x d y d z \\
\quad+\frac{1}{\operatorname{Re}_{2}} \int_{\Omega} e^{-z / H_{s}}\left|\nabla \pi_{z}\right|^{2} d x d y d z \\
\leq C\left(\|v\|_{6}^{4}+\left\|v_{z}\right\|_{2}^{2}\left\|\nabla v_{z}\right\|_{2}^{2}\right)\|\nabla \pi\|_{2}^{2}+C\|\pi\|_{2}^{2}+C\|\nabla \xi\|_{2}^{2} \\
\quad+C\left\|\pi_{z}\right\|_{2}^{2}+C\|\nabla v\|_{2}^{2}\|\Delta v\|_{2}^{2}\left\|\pi_{z}\right\|_{2}^{2}+C\|\pi\|^{2}\|\nabla v\|_{3}^{2} .
\end{aligned}
$$

Employing the uniform Gronwall inequality, we get

$$
\left\|\nabla v_{t}\right\|_{2}^{2} \leq \rho_{22},
$$

for any $t \geq T_{2}+11$.

Moreover, we have

$$
\begin{aligned}
& \frac{1}{\mathrm{Re}_{1}} \int_{t}^{t+1} \int_{\Omega} e^{-z / H_{s}}\left|\Delta v_{t}\right|^{2} d x d y d z d \tau \\
& \quad+\frac{1}{\operatorname{Re}_{2}} \int_{t}^{t+1} \int_{\Omega} e^{-z / H_{s}}\left|\nabla \partial_{z} v_{t}\right|^{2} d x d y d z d \tau \leq \rho_{23} .
\end{aligned}
$$

Similarly, we can also obtain

$$
\begin{gathered}
\left\|\nabla T_{t}\right\|_{2}^{2} \leq \rho_{24}, \\
\frac{1}{\operatorname{Re}_{1}} \int_{t}^{t+1} \int_{\Omega} e^{-z / H_{s}}\left|\Delta T_{t}\right|^{2} d x d y d z d \tau \\
+\frac{1}{\operatorname{Re}_{2}} \int_{t}^{t+1} \int_{\Omega} e^{-z / H_{s}}\left|\nabla \partial_{z} T_{t}\right|^{2} d x d y d z d \tau \leq \rho_{25},
\end{gathered}
$$

for any $t \geq T_{2}+12$.
3.15. $L^{2}$ Estimates of $\left(\partial_{z} v_{t}, \partial_{z} T_{t}\right)$. Denote that $\beta=\pi_{z}, \gamma=\xi_{z}$. It is clear that $\beta, \gamma$ satisfy the following equations obtained by differentiating (94) and (95) with respect to $z$, respectively,

$$
\begin{aligned}
\frac{\partial \beta}{\partial t} & +L_{1} \beta+\left(v_{z} \cdot \nabla\right) \pi+(v \cdot \nabla) \beta \\
& -\left(\int_{-h}^{z} e^{(z-\zeta) / H_{s}} \nabla \cdot v(x, y, \zeta, t) d \zeta\right) \frac{\partial \beta}{\partial z} \\
& -\frac{1}{H_{s}}\left(\int_{-h}^{z} e^{(z-\zeta) / H_{s}} \nabla \cdot v(x, y, \zeta, t) d \zeta\right) \beta \\
& -(\nabla \cdot v) \beta+f_{0} \vec{k} \times \beta+(\beta \cdot \nabla) v+(\pi \cdot \nabla) v_{z} \\
& -\left(\int_{-h}^{z} e^{(z-\zeta) / H_{s}} \nabla \cdot \pi(x, y, \zeta, t) d \zeta\right) \frac{\partial v_{z}}{\partial z} \\
& -\frac{1}{H_{s}}\left(\int_{-h}^{z} e^{(z-\zeta) / H_{s}} \nabla \cdot \pi(x, y, \zeta, t) d \zeta\right) \frac{\partial v}{\partial z} \\
& -(\nabla \cdot \pi) v_{z}+\frac{R}{H_{s}} \nabla \xi=0, \\
\frac{\partial \gamma}{\partial t} & +L_{2} \gamma+v_{z} \cdot \nabla \xi+v \cdot \nabla \gamma \\
& -\left(\int_{-h}^{z} e^{(z-\zeta) / H_{s}} \nabla \cdot v(x, y, \zeta, t) d \zeta\right) \frac{\partial \gamma}{\partial z} \\
& -(\nabla \cdot \pi) T_{z}-\frac{1}{H_{s}}\left(\int_{-h}^{z} e^{(z-\zeta) / H_{s}} \nabla \cdot v(x, y, \zeta, t) d \zeta\right) \gamma \\
& -(\nabla \cdot v) \gamma+\beta \cdot \nabla T+\pi \cdot \nabla T_{z} \\
& -\left(\int_{-h}^{z} e^{(z-\zeta) / H_{s}} \nabla \cdot \pi(x, y, \zeta, t) d \zeta\right) \frac{\partial T_{z}}{\partial z} \\
& \frac{1}{H_{s}}\left(\int_{-h}^{z} e^{(z-\zeta) / H_{s}} \nabla \cdot \pi(x, y, \zeta, t) d \zeta\right) \frac{\partial T}{\partial z}=0 \\
& (11)
\end{aligned}
$$

with the boundary conditions

$$
\begin{aligned}
\left.\beta\right|_{\Gamma_{u}}=0, \quad & \left.\beta\right|_{\Gamma_{b}}=0,\left.\quad \beta \cdot \vec{n}\right|_{\Gamma_{l}}=0, \\
& \frac{\partial \beta}{\partial \vec{n}} \times\left.\vec{n}\right|_{\Gamma_{l}}=0, \\
\left.\left(\frac{1}{R t_{2}} \gamma+\alpha \xi\right)\right|_{\Gamma_{u}}=0, & \left.\gamma\right|_{\Gamma_{b}}=0,\left.\quad \frac{\partial \gamma}{\partial \vec{n}}\right|_{\Gamma_{l}}=0 .
\end{aligned}
$$

Taking the $L^{2}$ inner product of (114) with $\beta$ and combining the boundary conditions (116), we have

$$
\begin{aligned}
\frac{1}{2} \frac{d}{d t}\|\beta\|_{2}^{2}+\|\beta\|^{2} \\
\leq \frac{1}{\operatorname{Re}_{2} H_{s}}\left\|\beta_{z}\right\|_{2}\|\beta\|_{2}+C\left\|v_{z}\right\|_{6}\|\nabla \pi\|_{3}\|\beta\|_{2} \\
\quad+C\|v\|_{6}\|\beta\|_{3}\|\nabla \beta\|_{2}+C\|\pi\|_{3}\left\|\nabla v_{z}\right\|_{2}\|\beta\|_{6} \\
\quad+C\|\nabla \pi\|_{3}\left\|\partial_{z} v_{z}\right\|_{2}\|\beta\|_{6}+C\|\xi\|_{2}\|\nabla \beta\|_{2} .
\end{aligned}
$$


Therefore, in view of the Young inequality, we obtain

$$
\begin{aligned}
\frac{d}{d t}\|\beta\|_{2}^{2} & +\|\beta\|^{2} \\
\leq & C\left(1+\|v\|_{6}^{4}\right)\|\beta\|_{2}^{2}+C\left\|v_{z}\right\|_{6}^{2}\|\nabla \pi\|_{3}^{2} \\
& +C\|\pi\|_{3}^{2}\left\|\nabla v_{z}\right\|_{2}^{2}+C\|\nabla \pi\|_{3}^{2}\left\|\partial_{z} v_{z}\right\|_{2}^{2}+C\|\xi\|_{2}^{2} .
\end{aligned}
$$

Employing the uniform Gronwall inequality, we get

$$
\left\|\partial_{z} v_{t}\right\|_{2}^{2} \leq \rho_{26}
$$

for any $t \geq T_{2}+13$.

Similarly, we can also obtain

$$
\int_{\Omega} e^{-z / H_{s}}\left|\partial_{z} T_{t}\right|^{2} d x d y d z+\alpha R t_{2} \int_{\Gamma_{u}}\left|T_{t}\right|^{2} d x d y \leq \rho_{27},
$$

for any $t \geq T_{2}+14$.

From (43), (48), (70), (74), (81), (85) and (108), we get the following results.

Theorem 7. Assume that $Q \in H^{1}(\Omega)$ and $\left(v_{0}, T_{0}\right) \in V$. Let $\{S(t)\}_{t \geq 0}$ be a semigroup generated by the initial boundary problem (14)-(18). Then, there exists an absorbing set in $\mathrm{H}^{2}$. That is, for any bounded subset $B$ of $V$, there exist a positive constant $\mathscr{R}_{1}$ and a positive time $\tau_{1}=\tau_{1, B}$ which depend on the norm of $B$, such that

$$
\begin{aligned}
\|(v(t), T(t))\|_{H^{2}}^{2} & =\left\|S(t)\left(v_{0}, T_{0}\right)\right\|_{H^{2}}^{2} \\
& =\|v(t)\|_{H^{2}}^{2}+\|T(t)\|_{H^{2}}^{2} \\
& \leq \mathscr{R}_{1},
\end{aligned}
$$

for any $t \geq \tau_{1}$.

Corollary 8. Assume that $Q \in H^{1}$ and $\left(v_{0}, T_{0}\right) \in V$. Let $\{S(t)\}_{t \geq 0}$ be a semigroup generated by the initial boundary problem (14)-(18). Then, there exist an absorbing set in $V$. That is, for any bounded subset $B$ of $V$, there exist a positive constant $\mathscr{R}_{2}$ and a positive time $\tau_{2}=\tau_{2, B}$, depending on the norm of $B$, such that

$$
\begin{aligned}
\|(v(t), T(t))\|_{V}^{2} & =\left\|S(t)\left(v_{0}, T_{0}\right)\right\|_{V}^{2} \\
& =\|v(t)\|_{V_{1}}^{2}+\|T(t)\|_{V_{2}}^{2} \\
& \leq \mathscr{R}_{2},
\end{aligned}
$$

for any $t \geq \tau_{2}$.

\section{The Existence of the Global Attractors}

In this section, we will recall some definitions and lemmas about the global attractor and prove the existence of the global attractor in $H^{2}$.
Definition 9 (see [23]). Let $X$ be a Banach space and $\{S(t)\}_{t \geq 0}$ a family operators on $X$. We say that $\{S(t)\}_{t \geq 0}$ is a norm-toweak continuous semigroup on $X$, if $\{S(t)\}_{t \geq 0}$ satisfies that

(i) $S(0)=$ Id (the identity),

(ii) $S(t) S(s)=S(t+s)$, for all $t, s \geq 0$,

(iii) $S\left(t_{n}\right) x_{n} \rightarrow S(t) x$ if $t_{n} \rightarrow t$ and $x_{n} \rightarrow x$ in $X$.

Lemma 10 (see [24]). Let $X, Y$ be two Banach spaces and $X^{*}$, $Y^{*}$ be the dual spaces of $X, Y$, respectively. If $X$ is dense in $Y$, the injection $i: X \rightarrow Y$ is continuous and its adjoint $i^{*}: Y^{*} \rightarrow X^{*}$ is dense. Let $\{S(t)\}_{t \geq 0}$ be a semigroup on $X$ and $Y$, respectively, and assume furthermore that $\{S(t)\}_{t \geq 0}$ is continuous or weak continuous on $Y$. Then, $\{S(t)\}_{t \geq 0}$ is a normto-weak continuous semigroup on $X$ if and only if $\{S(t)\}_{t \geq 0}$ maps compact subsets of $\mathbb{R}^{+} \times X$ into bounded sets of $X$.

Definition 11 (see [20]). Let $X$ be a Banach space and let $\{S(t)\}_{t \geq 0}$ be a continuous semigroup on $X$. A subset $\mathscr{A}$ in $X$ is called a global attractor if and only if

(1) $\mathscr{A}$ is invariant; that is, $S(t) \mathscr{A}=\mathscr{A}$ for all $t \geq 0$,

(2) $\mathscr{A}$ is compact in $X$,

(3) $\mathscr{A}$ attracts each bounded subset $B$ in $X$.

Definition 12 (see [20]). Let $\{S(t)\}_{t \geq 0}$ be a semigroup on Banach space $X .\{S(t)\}_{t \geq 0}$ is called $(X, Z)$ uniformly compact, if for any bounded (in $X$ ) set $B \subset X$, there exists $t_{0}=t_{0}(B)$, such that $\cup_{t \geq t_{0}} S(t) B$ is relatively compact in $Z$.

Lemma 13 (see $[20,24])$. Let $X$ be a Banach space and $\{S(t)\}_{t \geq 0}$ a $C^{0}$ semigroup on $X$. Then $\{S(t)\}_{t \geq 0}$ has a global attractor $\mathscr{A}$ in $X$ provided that the following conditions hold true:

(1) $\{S(t)\}_{t \geq 0}$ has a bounded absorbing set $B_{0}$ in $X$,

(2) $\{S(t)\}_{t \geq 0}$ is uniformly compact.

Lemma 14 (see $[20,24,25])$. Let $\{S(t)\}_{t \geq 0}$ be a norm-to-weak continuous semigroup such that $\{S(t)\}_{t \geq 0}$ is uniformly compact. If there exists an absorbing set $B$, then $\{S(t)\}_{t \geq 0}$ has a global attractor $\mathscr{A}$ and

$$
\mathscr{A}=\bigcap_{s \geq 0} \overline{\bigcup_{t \geq s} S(t) B}
$$

By virtue of the Rellich-Kondrachov theorem, we obtain the following.

Corollary 15. Assume that $Q \in H^{1},\left(v_{0}, T_{0}\right) \in V$. Then, the semigroup $\{S(t)\}_{t \geq 0}$ corresponding to (14)-(18) has a $(V, V)$ global attractor $\mathscr{A}_{V}$.

To prove the existence of the $\left(V, H^{3}\right)$-absorbing set, we need the following lemma. 
Lemma 16 (see [7]). Let $v \in V_{1}, \Phi_{s} \in L^{2}(M)$ be a solution to the following Stokes problem:

$$
\begin{gathered}
L_{1} v+\nabla \Phi_{s}=g, \\
\int_{-h}^{0} \nabla \cdot v(x, y, z) d z=0, \\
\left.\frac{\partial v}{\partial z}\right|_{z=-h}=0,\left.\quad \frac{\partial v}{\partial z}\right|_{z=0}=0 .
\end{gathered}
$$

If $g \in\left(W^{m, \alpha}(\Omega)\right)^{2}, 1<\alpha<\infty, m \geq 0$, then

$$
v \in V_{1} \cap\left(W^{m+2, \alpha}(\Omega)\right)^{2}, \quad \Phi_{s} \in W^{m+1, \alpha}(M) .
$$

Next, we will prove the existence of absorbing set in $H^{3}$ by the use of Lemma 16, which implies the existence of $\left(V, H^{2}\right)$ global attractor of the initial boundary problem (14)-(18).

Theorem 17. Assume that $Q \in H^{1}$ and $\left(v_{0}, T_{0}\right) \in V$. Let $\{S(t)\}_{t \geq 0}$ be a semigroup generated by the initial boundary problem (14)-(18). Then, there exists an absorbing set in $H^{3}$. That is, for any bounded subset $B$ of $V$, there exists a positive constant $\mathscr{R}_{3}$ depending on the norm of $B$, such that

$$
\begin{aligned}
\|(v(t), T(t))\|_{H^{3}}^{2} & =\left\|S(t)\left(v_{0}, T_{0}\right)\right\|_{H^{3}}^{2} \\
& =\|v(t)\|_{H^{3}}^{2}+\|T(t)\|_{H^{3}}^{2} \\
& \leq \mathscr{R}_{3},
\end{aligned}
$$

for any $t \geq \tau_{1}$, where $\tau_{1}$ is specified in Theorem 7 .

Proof. From the results of a priori estimates in the previous section, we know that there exists a strong solution $(v, T)$ to (14)-(18), which satisfies for any $\mathscr{T}>\tau_{1}, v_{z} \in$ $L^{\infty}\left(\tau_{1}, \mathscr{T} ; L^{6}\right), T_{z} \in L^{\infty}\left(\tau_{1}, \mathscr{T} ; L^{6}\right), v_{t} \in L^{\infty}\left(\tau_{1}, \mathscr{T} ; V_{1}\right), T_{t} \in$ $L^{\infty}\left(\tau_{1}, \mathscr{T} ; V_{2}\right),(v, T) \in L^{\infty}\left(\tau_{1}, \mathscr{T} ; H^{2}\right)$, where $\tau_{1}$ is specified in Theorem 7. Then,

$$
L_{1} v+\nabla \Phi_{s}=g
$$

where

$$
\begin{aligned}
-g= & \frac{\partial v}{\partial t}+(v \cdot \nabla) v-\left(\int_{-h}^{z} e^{(z-\zeta) / H_{s}} \nabla \cdot v(x, y, \zeta, t) d \zeta\right) \frac{\partial v}{\partial z} \\
& +\frac{R}{H_{s}} \int_{0}^{z} \nabla T(x, y, \zeta, t) d \zeta+f_{0} \vec{k} \times v .
\end{aligned}
$$

Since $v \in L^{\infty}\left(\tau_{1}, \mathscr{T} ; H^{2}\right)$ and $v_{z} \in L^{\infty}\left(\tau_{1}, \mathscr{T} ; L^{6}\right)$, for any $u \in$ $L^{3 / 2}$, we have

$$
\begin{gathered}
\left|\int_{\Omega} e^{-z / H_{s}}\left(\int_{-h}^{z} e^{(z-\zeta) / H_{s}} \nabla \cdot v(x, y, \zeta, t) d \zeta\right) \frac{\partial v}{\partial z} \cdot u d x d y d z\right| \\
\leq C\|\nabla v\|_{6}\left\|v_{z}\right\|\left\|_{6}\right\| u \|_{3 / 2},
\end{gathered}
$$

which implies that

$$
\left(\int_{-h}^{z} e^{(z-\zeta) / H_{s}} \nabla \cdot v(x, y, \zeta, t) d \zeta\right) \frac{\partial v}{\partial z} \in L^{\infty}\left(\tau_{1}, \mathscr{T} ; L^{3}\right) .
$$

Since $(v, T)$ is a strong solution to (14)-(18) and $v_{t} \in$ $L^{\infty}\left(\tau_{1}, \mathscr{T} ; V_{1}\right)$, it is clear that $\partial v / \partial t+(v \cdot \nabla) v-\left(R / H_{s}\right) \int_{0}^{z} \nabla T(x$, $y, \zeta, t) d \zeta+f_{0} \vec{k} \times v \in L^{\infty}\left(\tau_{1}, \mathscr{T} ; L^{3}\right)$. Thus,

$$
g \in L^{\infty}\left(\tau_{1}, \mathscr{T} ; L^{3}\right) .
$$

By the use of Lemma 16, we get

$$
v \in L^{\infty}\left(\tau_{1}, \mathscr{T} ; V_{1} \cap\left(W^{2,3}(\Omega)\right)^{2}\right) .
$$

Using the Sobolev embedding theorem $\left(W^{2,3}(\Omega)\right)^{2} \quad C$ $\left(W^{1,12}(\Omega)\right)^{2}$, we know that

$$
v \in L^{\infty}\left(\tau_{1}, \mathscr{T} ; V_{1} \cap\left(W^{1,12}(\Omega)\right)^{2}\right) .
$$

Now, since $v \in L^{\infty}\left(\tau_{1}, \mathscr{T} ; V_{1} \cap\left(W^{1,12}(\Omega)\right)^{2}\right)$ and $v_{z} \in L^{\infty}\left(\tau_{1}\right.$, $\left.\mathscr{T} ; L^{6}\right)$, for any $u \in L^{4 / 3}$, we have

$$
\begin{gathered}
\left|\int_{\Omega} e^{-z / H_{s}}\left(\int_{-h}^{z} e^{(z-\zeta) / H_{s}} \nabla \cdot v(x, y, \zeta, t) d \zeta\right) \frac{\partial v}{\partial z} \cdot u d x d y d z\right| \\
\leq C\|\nabla v\|_{12}\left\|v_{z}\right\|_{6}\|u\|_{4 / 3},
\end{gathered}
$$

which implies that

$$
\left(\int_{-h}^{z} e^{(z-\zeta) / H_{s}} \nabla \cdot v(x, y, \zeta, t) d \zeta\right) \frac{\partial v}{\partial z} \in L^{\infty}\left(\tau_{1}, \mathscr{T} ; L^{4}\right) .
$$

Therefore, we find that

$$
g \in L^{\infty}\left(\tau_{1}, \mathscr{T} ; L^{4}\right) .
$$

From Lemma 16, we know that

$$
v \in L^{\infty}\left(\tau_{1}, \mathscr{T} ; V_{1} \cap\left(W^{2,4}(\Omega)\right)^{2}\right),
$$

which implies that

$$
\left(\int_{-h}^{z} e^{(z-\xi) / H_{s}} \nabla \cdot v(x, y, \zeta, t) d \zeta\right) \frac{\partial v}{\partial z} \in L^{\infty}\left(\tau_{1}, \mathscr{T} ; H^{1}\right) .
$$

Since $v_{t} \in L^{\infty}\left(\tau_{1}, \mathscr{T} ; V_{1}\right)$ and $v \in L^{\infty}\left(\tau_{1}, \mathscr{T} ; V_{1} \cap\left(W^{2,4}(\Omega)\right)^{2}\right)$, it is clear that $\partial v / \partial t+(v \cdot \nabla) v-\left(R / H_{s}\right) \int_{0}^{z} \nabla T(x, y, \zeta, t) d \zeta+$ $f_{0} \vec{k} \times v \in L^{\infty}\left(\tau_{1}, \mathscr{T} ; H^{1}\right)$. Thus,

$$
g \in L^{\infty}\left(\tau_{1}, \mathscr{T} ; H^{1}\right) .
$$

From Lemma 16, we deduce that

$$
v \in L^{\infty}\left(\tau_{1}, \mathscr{T} ; V_{1} \cap H^{3}\right) .
$$

Employing the elliptic regularity theory, we obtain

$$
T \in L^{\infty}\left(\tau_{1}, \mathscr{T} ; V_{2} \cap H^{3}\right) .
$$

This complete the proof of Theorem 17. 
Since $H^{2} \subset V$ is compact, from Theorem 7, we know that the semigroup generated by (14)-(18) is compact in $V$. From Theorem 7 and Lemma 10, we deduce that the semigroup generated by (14)-(18) maps a compact set of $H^{2}$ to be a bounded set of $H^{2}$; that is, the semigroup generated by (14)-(18) is norm to weak continuous in $H^{2}$. Furthermore, $H^{3} \subset H^{2}$ compactly, and from Theorem 7 and Lemma 13, we immediately get the following result.

Theorem 18. Assume that $Q \in H^{1}(\Omega),\left(v_{0}, T_{0}\right) \in V$. Then, the semigroup $\{S(t)\}_{t \geq 0}$ corresponding to (14)-(18) has a $\left(V, H^{2}\right)$-global attractor $\mathscr{A}$.

By using the Rellich-Kondrachov theorem, we can obtain the existence of the $\left(V, H^{2+\delta}\right)$-global attractor for $\delta \in[0,1)$.

\section{Appendix}

\section{Proof of Proposition 2}

If this conclusion is not true, then for each natural number $n$, there exists $v_{n} \in V_{1}$ such that

$$
\left\|v_{n}\right\|_{2}^{2}=1, \quad\left\|v_{n}\right\|^{2} \leq \frac{1}{n} .
$$

Let

$$
\left\|v_{n}\right\|_{H^{1}}^{2}=\left\|v_{n}\right\|_{2}^{2}+\left\|v_{n}\right\|^{2} .
$$

Hence,

$$
\left\|v_{n}\right\|_{H^{1}}^{2} \leq 1+\frac{1}{n} \leq 2,
$$

which implies that there exists a subsequence of $\left\{v_{n}\right\}_{n \geq 1}$ (still denoted by $\left\{v_{n}\right\}_{n \geq 1}$ ) such that

$$
\begin{gathered}
v_{n} \longrightarrow v, \quad \text { weakly in } H^{1}, \\
v_{n} \longrightarrow v, \quad \text { strongly in } L^{2} .
\end{gathered}
$$

Therefore, we have

$$
\|v\|_{2}^{2}=1, \quad\|v\|_{H^{1}}^{2} \leq \liminf _{n \rightarrow \infty}\left\|v_{n}\right\|_{H^{1}}^{2}=1,
$$

which implies that

$$
\|v\|^{2}=0
$$

Combining $\|v\|^{2}=0$ with $v \cdot \vec{n}=0$, we obtain

$$
v=0,
$$

this is a contradiction.

\section{References}

[1] J.-L. Lions, R. Temam, and S. H. Wang, "New formulations of the primitive equations of atmosphere and applications," Nonlinearity, vol. 5, no. 2, pp. 237-288, 1992.
[2] J. Pedlosky, Geophysical Fluid Dynamics, Springer, New York, NY, USA, 1987.

[3] G. K. Vallis, Atmospheric and Oceanic Fluid Dynamics: Fundamentals and Large-Scale Circulation, Cambridge University Press, Cambridge, UK, 2006.

[4] C. Cao and E. S. Titi, "Global well-posedness of the threedimensional viscous primitive equations of large scale ocean and atmosphere dynamics," Annals of Mathematics, vol. 166, no. 1, pp. 245-267, 2007.

[5] B. D. Ewald and R. Témam, "Maximum principles for the primitive equations of the atmosphere," Discrete and Continuous Dynamical Systems, vol. 7, no. 2, pp. 343-362, 2001.

[6] D. W. Huang and B. L. Guo, "On the existence of atmospheric attractors," Science in China, Series D, vol. 51, no. 3, pp. 469-480, 2008.

[7] B. Guo and D. Huang, "On the 3D viscous primitive equations of the large-scale atmosphere," Acta Mathematica Scientia. Series $B$, vol. 29, no. 4, pp. 846-866, 2009.

[8] B. Guo and D. Huang, "Existence of weak solutions and trajectory attractors for the moist atmospheric equations in geophysics," Journal of Mathematical Physics, vol. 47, no. 8, p. 083508, 23, 2006.

[9] C. Hu, R. Temam, and M. Ziane, "The primitive equations on the large scale ocean under the small depth hypothesis," Discrete and Continuous Dynamical Systems. Series A, vol. 9, no. 1, pp. 97-131, 2003.

[10] C. Hu, "Asymptotic analysis of the primitive equations under the small depth assumption," Nonlinear Analysis: Theory, Methods \& Applications, vol. 61, no. 3, pp. 425-460, 2005.

[11] J.-L. Lions, R. Temam, and S. H. Wang, "On the equations of the large-scale ocean," Nonlinearity, vol. 5, no. 5, pp. 1007-1053, 1992.

[12] T. Tachim Medjo, "On the uniqueness of $z$-weak solutions of the three-dimensional primitive equations of the ocean," Nonlinear Analysis: Real World Applications, vol. 11, no. 3, pp. 1413-1421, 2010.

[13] M. Petcu, R. Temam, and D. Wirosoetisno, "Existence and regularity results for the primitive equations in two space dimensions," Communications on Pure and Applied Analysis, vol. 3, no. 1, pp. 115-131, 2004.

[14] F. Guillén-González, N. Masmoudi, and M. A. RodríguezBellido, "Anisotropic estimates and strong solutions of the primitive equations," Differential and Integral Equations, vol. 14, no. 11, pp. 1381-1408, 2001.

[15] R. Temam and M. Ziane, "Some mathematical problems in geophysical fluid dynamics," in Handbook of Mathematical Fluid Dynamics. Vol. III, pp. 535-657, North-Holland, Amsterdam, 2004.

[16] G. M. Kobelkov, "Existence of a solution 'in the large' for the 3D large-scale ocean dynamics equations," Comptes Rendus Mathematique, vol. 343, no. 4, pp. 283-286, 2006.

[17] N. Ju, "The global attractor for the solutions to the 3D viscous primitive equations," Discrete and Continuous Dynamical Systems. Series A, vol. 17, no. 1, pp. 159-179, 2007.

[18] B. You, C. K. Zhong, and G. C. Yue, "Global attractor for the three dimensional viscous primitive equations oflarge-scale ocean and atmosphere dynamics," submitted.

[19] R. A. Adams, Sobolev Spaces, vol. 65 of Pure and Applied Mathematics, Academic Press, New York, NY, USA, 1975.

[20] R. Temam, Infinite-Dimensional Dynamical Systems in Mechanics and Physics, vol. 68 of Applied Mathematical Sciences, Springer, New York, 2nd edition, 1997. 
[21] R. Temam, Navier-Stokes Equations Theory and Numerical Analysis, vol. 2 of Studies in Mathematics and Its Applications, North-Holland, Amsterdam, The Netherlands, 1979.

[22] R. Temam, Navier-Stokes Equations and Nonlinear Functional Analysis, vol. 41 of CBMS-NSF Regional Conference Series in Applied Mathematics, SIAM, Philadelphia, Pa, USA, 1983.

[23] Q. Ma, S. Wang, and C. Zhong, "Necessary and sufficient conditions for the existence of global attractors for semigroups and applications," Indiana University Mathematics Journal, vol. 51, no. 6, pp. 1541-1559, 2002.

[24] C.-K. Zhong, M.-H. Yang, and C.-Y. Sun, "The existence of global attractors for the norm-to-weak continuous semigroup and application to the nonlinear reaction-diffusion equations," Journal of Differential Equations, vol. 223, no. 2, pp. 367-399, 2006.

[25] A. V. Babin and M. I. Vishik, Attractors of Evolution Equations, vol. 25 of Studies in Mathematics and Its Applications, NorthHolland, Amsterdam, The Netherlands, 1992. 


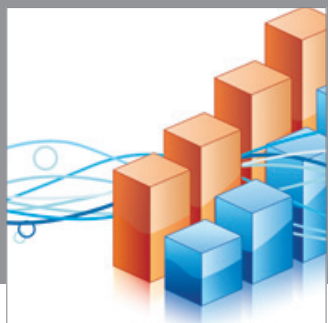

Advances in

Operations Research

mansans

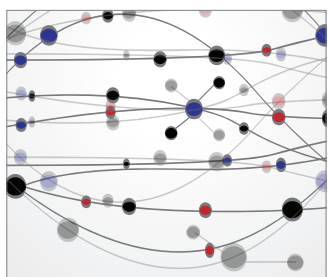

The Scientific World Journal
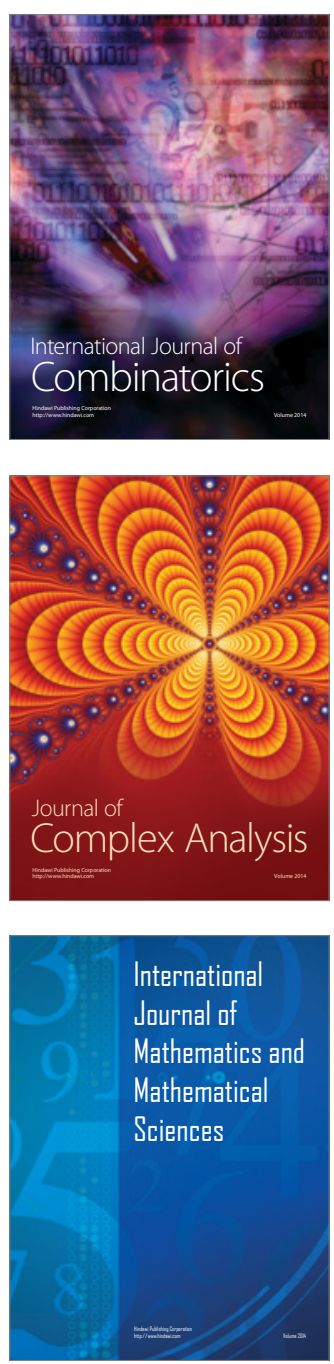
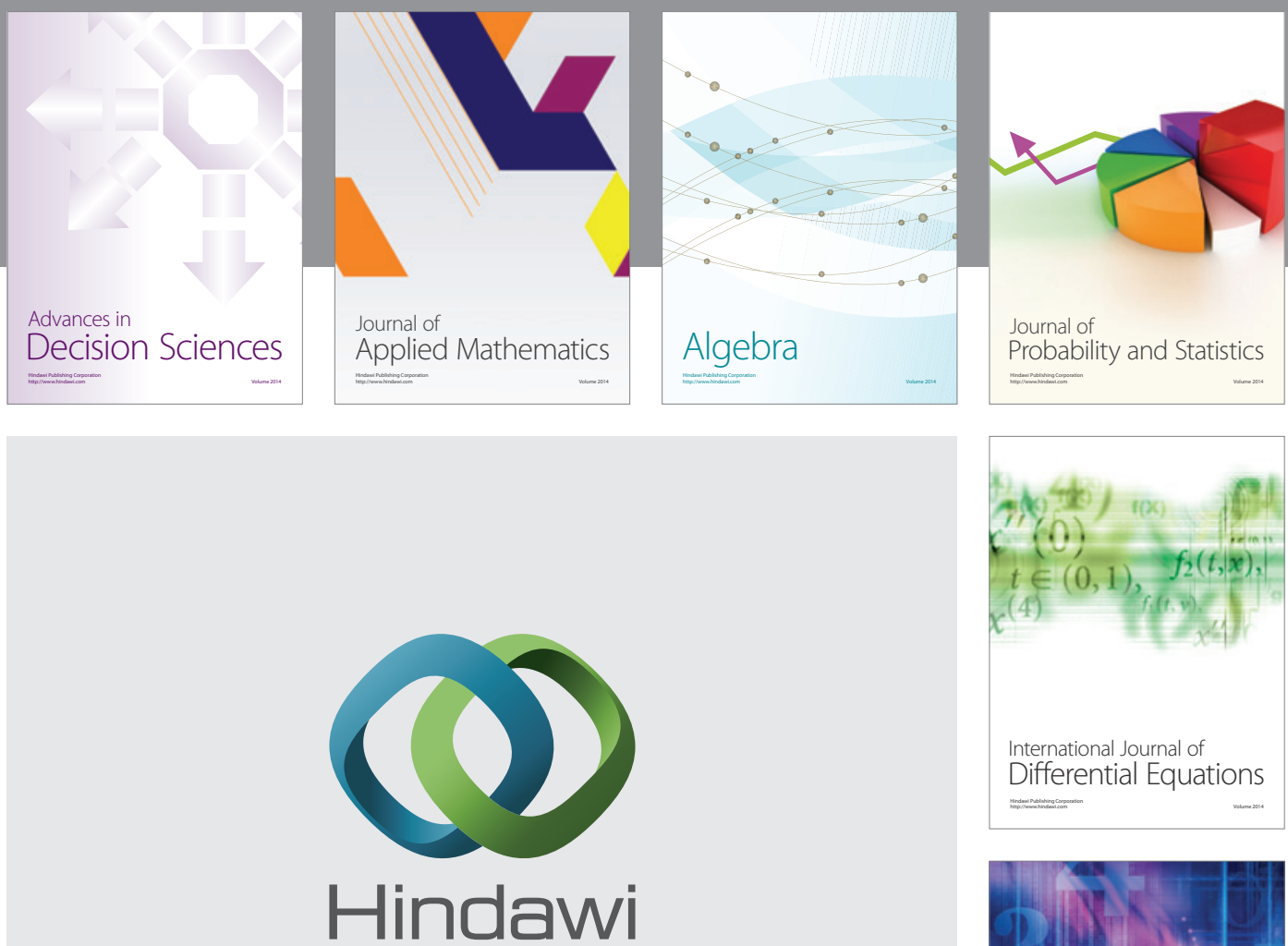

Submit your manuscripts at http://www.hindawi.com
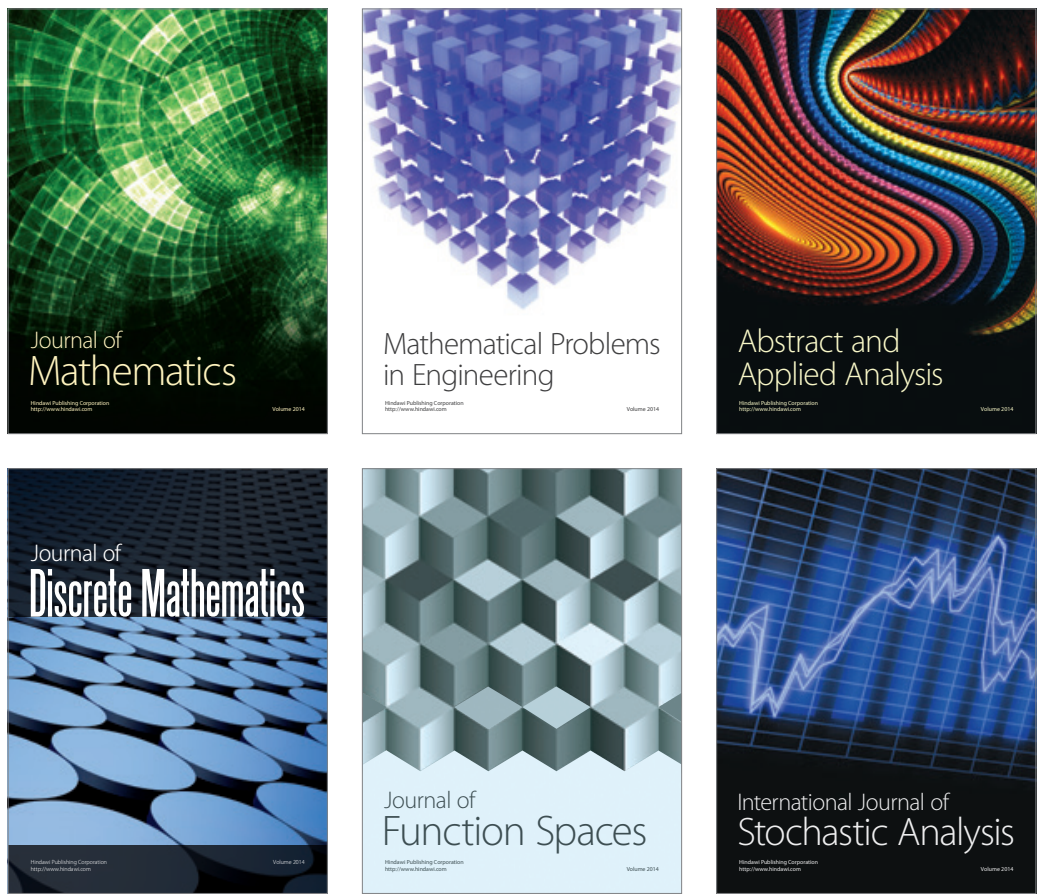

Journal of

Function Spaces

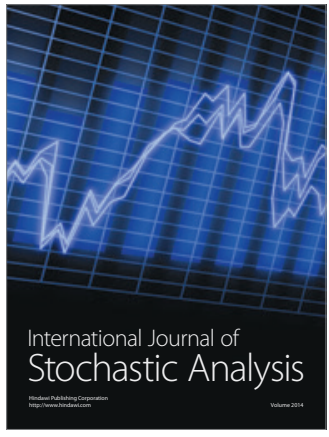

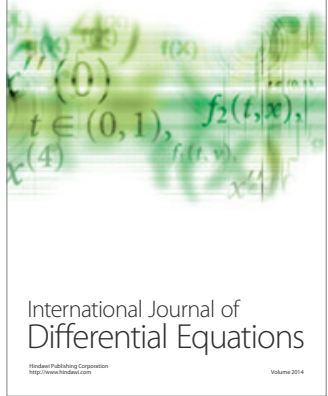
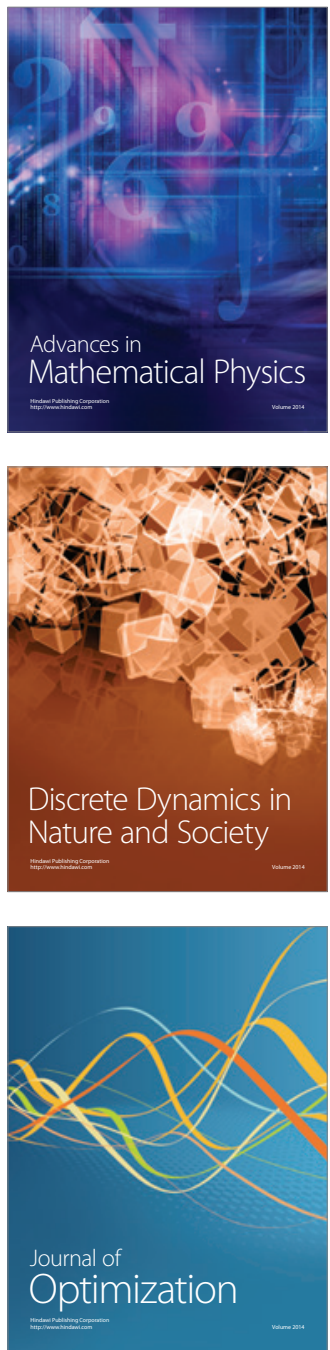